CAE Working Paper \#05-16

General Equilibrium with Nonconvexities, Sunspots, and Money

by

\author{
Guillaume Rocheteau \\ Peter Rupert \\ Karl Shell \\ and \\ Randall Wright
}

November 2005 


\title{
General Equilibrium with Nonconvexities, Sunspots, and Money*
}

\author{
Guillaume Rocheteau Peter Rupert Karl Shell \\ Randall Wright
}

November 28, 2005

\begin{abstract}
We study general equilibrium with nonconvexities. In these economies there exist sunspot equilibria without the usual assumptions needed in convex economies, and they have good welfare properties. Moreover, in these equilibria, agents act as if they have quasi-linear utility. Hence wealth effects vanish. We use this to construct a new model of monetary exchange. As in Lagos-Wright, trade occurs in both centralized and decentralized markets, but while that model requires quasilinearity, we have general preferences. Given our specification looks much like the textbook Arrow-Debreu model, we think this constitutes progress on the classic problem of integrating money and general equilibrium theory. We also use the model to discuss another classic issue: the relation between inflation and unemployment.
\end{abstract}

${ }^{*}$ Rocheteau and Rupert: Federal Reserve Bank of Cleveland; Shell: Cornell University; Wright: University of Pennsylvania. For their input we thank Borogan Aruoba, Felix Kubler, and seminar participants at the Cleveland Fed, Penn, Penn State, Princeton, LSE, Cornell, Notre Dame, Simon Fraser, Essex, and the Canadian Economic Theory meetings at UBC. We thank the NSF for research support. In addition to the usual disclaimer, the views expressed here are those of the authors and not necessarily those of the Federal Reserve Bank of Cleveland or the Federal Reserve System. 


\section{Introduction}

We study economies with nonconvexities, and in particular with some indivisible goods, with several goals in mind. First, extending Shell and Wright (1993) we show that in the presence of indivisibilities there exist sunspot equilibria without the usual assumptions needed to generate such equilibria in convex economies, and that these equilibria have good welfare properties because sunspots allow convexification similar to the way lotteries work in the indivisible labor economy of Rogerson (1988). ${ }^{1}$ Second, we emphasize something not appreciated in the existing literature on sunspots, lotteries and nonconvexities: in these economies, as long as agents choose interior solutions, in a sense to be made precise, they act as if they have quasi-linear preferences.

It is true that it has been noted previously of the Rogerson model that, when labor is indivisible, under certain additional assumptions that include additive separability between consumption and leisure, agents act as if utility is linear in leisure. But the result is far more general. The fact that for quite general specifications agents act as if they have quasi-linear preferences is useful for a variety of reasons. For one thing, it means that for the divisible goods in the economy, wealth effects vanish. This has many implications, including the law of demand (the demand for each of the divisible goods is unambiguously decreasing in its own price). Here we will emphasize the usefulness of the results for monetary theory, as we now explain.

\footnotetext{
${ }^{1} \mathrm{~A}$ sunspot equilibrium is one in which extrinsic uncertainty (a random variable with no impact on preferences, endowments, or technologies) affects the allocation. In convex economies, sunspot equilibria are necessarily inefficient, because random allocations are dominated by the average allocation. When some goods are indivisible, however, the average may not be feasible. For some recent papers on nonconvexities, lotteries, and sunspots, in addition to those cited here, see the 2002 special issue of JET
} 
A novel model of monetary exchange with microfoundations based on search theory has been developed recently by Lagos and Wright (2005), hereafter LW. The LW model is tractable because gets around the problem of having to keep track of the distribution of money holdings. It works by giving agents periodic access to centralized markets where they can adjust their cash positions by buying and selling other goods, in addition to the decentralized markets where money is essential. If agents have quasi-linear utility, given interior solutions, they all take the same amount of money out of the centralized market, and hence the distribution in the decentralized market is degenerate. This makes the framework quite easy to analyze, and hence allows one to extend and apply it in a number of ways. ${ }^{2}$

Although for some questions one would obviously like to have an endogenous nondegenerate distribution of money holdings, it is useful to have a benchmark without this complication, and to this extent the LW model is interesting. One might object, however, that quasi-linear utility is very special. Our results show that one does not actually need quasi-linearity: for general preferences, as long as some goods are indivisible, and again given interior solutions, agents act as if they have quasi-linear utility in the sense that they all take the same amount of money out of the centralized market. Thus, we provide an alternative set of assumptions that leads to a very simple model of monetary exchange with explicit microfoundations.

\footnotetext{
${ }^{2} \mathrm{LW}$ provide examples and references to other applications. An alternative approach is provided by Shi (1997); Faig (2004) tries to integrate the two models, and gives some results related to those derived below. For models that are much less tractable, precisely because one has to keep track of the relevant distribution, see Green and Zhou (1998), Zhou (1999), Molico (1999), Camera and Corbae (1999), Taber and Wallace (1999), or Zhu $(2003,2005)$. Earlier search-based models, such as Kiyotaki and Wright $(1989,1993)$, Aiyagari and Wallace (1991), Shi (1995), or Trejos and Wright (1995), were also very simple, but only because they avoided the issue by assuming agents could only hold $m \in$ $\{0,1\}$ units of money.
} 
We make an effort to describe the centralized market in the model in a fairly general way - we have few restrictions on endowments, tastes, or technologies other than those in standard general equilibrium (GE) theory, except that we have some indivisible goods. This generality is adopted because it entails little cost for what we do, and also because it indicates that modern monetary theory is not as special as one might think based on previous presentations. In earlier discussions of the LW model, e.g., the centralized market typically has a single consumption good, consumers are homogeneous in terms of preferences and endowments, firms (if there are firms at all) are homogeneous, and so on. We show that most of these special assumptions are completely unnecessary.

Indeed, our centralized market looks very much like the textbook ArrowDebreu model with state-contingent commodities (Debreu 1959, ch. 7). Thus we can appeal to some standard results in GE. Moreover, given we combine this specification with a micro-based monetary model, one might say that we make a little progress on the classic problem of integrating of money and GE theory. Interestingly, we think, progress here comes not from a Procrustean effort to force money into GE, but from bringing GE into monetary theory. Finally, under a common interpretation in macro that labor is indivisible, the model generates equilibrium unemployment. ${ }^{3}$ And since it is a monetary model, we can use it to discuss another classic issue: the relation between inflation and unemployment. We show that the model generates a longrun Phillips curve that is either upward or downward sloping, depending on preferences, in a simple and natural way.

\footnotetext{
${ }^{3}$ In addition to Rogerson (1988), a sample of well-known papers in macroeconomics adopting the indivisible labor assumption includes Hansen (1985), Cooley and Hansen (1989), and Christiano and Eichenbaum (1992).
} 
The rest of the paper is organized as follows. In Section 2 we discuss indivisibilities and sunspots in GE, without money. We show that agents not at a corner solution act as if they have quasi-linear utility, in the sense that their demands for divisible goods are independent of wealth, and their indirect utility functions are linear in wealth. We also discuss conditions to guarantee interiority. In Section 3 we consider monetary economies. We begin with brief review of LW, then present our alternative model, and compare results. In Section 4 we discuss the relation between inflation and unemployment. In Section 5 we conclude. Some technical results are relegated to the Appendix.

\section{GE with Nonconvexities}

\subsection{Equilibrium: Definition}

We begin with a static GE model. ${ }^{4}$ There is a measure space $(I, \Omega, \alpha)$ of consumers, where $I=[0,1], \Omega$ a $\sigma$-algebra of subsets of $I$, and $\alpha$ the uniform distribution over $I .^{5}$ There are $K$ firms indexed by $k=1, \ldots K$. There are $J+1$ commodities: $J$ standard consumption goods indexed by $j=1, \ldots J$, and one indivisible good. We call the indivisible good leisure, following some examples in macro, although this label means little for now.

\footnotetext{
${ }^{4} \mathrm{By}$ static, we do not mean the economy is timelss, since as usual one can interpret goods as indexed by dates. We simply mean that there is a single market that convenes before any production and consumption take place. Later we consider sequential-market models.

${ }^{5}$ We take this specification from Aumann (1964,1966), who first studied equilibrium with a continuum of agents. We do not actually need a continuum here, but it is adopted because in the monetary models discussed below, as in much of the literature, when combined with random matching it generates anonymity. It is worth mentioning that we could get away with a finite number of agents (for the GE results, and also for the monetary results as long as we have some other way to motivate anonymity) because we use sunspots as opposed to lotteries; the latter generally need the law of large numbers while the former do not (Shell and Wright 1993; Garratt, Keister and Shell 2004).
} 
By saying leisure is indivisible, we mean that it must either be consumed in a single unit or not at all.

Agent $i$ starts with 1 unit of leisure, and an arbitrary endowment of the other goods $\mathbf{e}^{i} \in \mathbb{R}_{+}^{J}$, where $\mathbf{e}^{i}: I \rightarrow \mathbb{R}_{+}^{J}$ is $I$-measurable and $\overline{\mathbf{e}}=\int \mathbf{e}^{i} d i$. Consumer $i$ has preferences represented by a von Neumann-Morgenstern utility function $U^{i}(\mathbf{c}, h)$, where $\mathbf{c} \in \mathbb{R}_{+}^{J}$ is consumption and $h \in\{0,1\}$ is labor, which equals 1 minus leisure. The consumption set for each agent is denoted $C=\mathbb{R}_{+}^{J} \times\{0,1\}$. We assume $U^{i}$ is twice continuously differentiable, strictly increasing in c, strictly decreasing in $h$, and strictly concave. Merely to ease the presentation, we assume $U_{j}(\mathbf{c}, h) \rightarrow \infty$ as $c_{j} \rightarrow 0$ for all $j$, where $U_{j}$ is the partial derivative with respect to $c_{j}$, to guarantee $c_{j}>0$.

Consumption goods are produced by firms using labor as the only input. Firm $k$ has a technology represented by production function $\mathbf{f}^{k}\left(n^{k}\right)=$ $\left[f_{1}^{k}\left(n^{k}\right), \ldots f_{J}^{k}\left(n^{k}\right)\right] \in \mathbb{R}_{+}^{J}$, where $f_{j}^{k}\left(n^{k}\right)$ is output of good $j$. Assume $\mathbf{f}^{k}$ is continuously differentiable, increasing and concave. It is possible that $f_{j}^{k}\left(n^{k}\right)=0$ for all $n$ for some $j$ - i.e. each firm $k$ does not necessarily produce every good - but for any good it does produce $f_{j}^{k}$ is strictly increasing and concave. Profit for firm $k$ (defined below) is $\Pi^{k}$, and the share of $\Pi^{k}$ paid to consumer $i$ is $\eta_{k}^{i} \in \mathbb{R}_{+}$where $\int \eta_{k}^{i} d i=1$. Thus total dividend income for consumer $i$ is $\Delta^{i}=\sum_{k} \eta_{k}^{i} \Pi^{k}$.

Consumers are generally heterogenous, but for simplicity we assume there are only a finite number of types $T$; that is, $I=\cup_{\tau=1}^{T} I_{\tau}$, where $U^{i}=U^{\tau}, e^{i}=e^{\tau}$ and $\eta_{k}^{i}=\eta_{k}^{\tau}$ for all $i \in I_{\tau}$. Also, for simplicity, there is no intrinsic uncertainty: all of the fundamentals are deterministic. However, there is extrinsic uncertainty, represented by the probability space $(S, \Sigma, \pi)$, where $S=[0,1]$ is the set of states representing "sunspot activity," $\Sigma$ the 
Borel sets on $S$, and $\pi$ the uniform distribution over $S$. For what we do here, the choice of a uniform distribution is without loss in generality (Garratt, Keister, Qin and Shell 2002). Although the realization of $s \in S$ does not affect preferences, technology or endowments, in principle it could still affect individual's behavior.

Given indivisible goods, having allocations potentially depend on extrinsic uncertainty allows a certain convexification, which can lead to efficiency gains over nonrandomized allocations. ${ }^{6}$ Following Shell and Wright (1993), we formalize this by assuming complete Arrow-Debreu markets in sunspotstate-contingent commodities. Thus, the commodity space is the set of $\pi$ measurable functions from $S$ into $C$. Similarly, $n^{k}(s)$ is firm $k^{\prime}$ s employment rule, a $\pi$-measurable function from $S$ into $\mathbb{R}_{+}$. Let $\mathbf{p}(s)=\left[p_{1}(s), \ldots p_{J}(s)\right] \in$ $\mathbb{R}_{+}^{J}$ be the price vector of consumption goods and $w(s) \in \mathbb{R}_{+}$the price of labor in state $s .{ }^{7}$ For all $\tilde{S} \subset S, \int_{\tilde{S}} p_{j}(s) d s$ is the cost of a unit of good $j$ if event $\tilde{S}$ occurs. Let $\left[\mathbf{c}^{i}(s), h^{i}(s)\right]$ list a point in commodity space for every consumer $i$, and $\left[n^{k}(s)\right]$ an employment rule for every firm $k$.

Definition 1 An equilibrium is a list $\left\{\left[\mathbf{c}^{i}(s), h^{i}(s)\right],\left[n^{k}(s)\right],[\mathbf{p}(s), w(s)]\right\}$ satisfying:

(i) given $[\mathbf{p}(s), w(s)], \forall i\left[\mathbf{c}^{i}(s), h^{i}(s)\right]$ solves

$$
W^{i}=\max _{\mathbf{c}^{i}(s), h^{i}(s)} \int_{S} U^{i}\left[\mathbf{c}^{i}(s), h^{i}(s)\right] d s
$$

\footnotetext{
${ }^{6}$ One can define competitive equilibrium without sunspots in the model. By the First Welfare Theorem, which does not require convexity, if it exists such an equilibrium is Pareto optimal within the set of nonrandomized allocations. It is easy to provide robust examples, however, where it is Pareto dominated by randomized allocations, including sunspot equilibrium allocations (see e.g. Shell and Wright 1993).

${ }^{7}$ We restrict attention to price systems that have an inner-product representation (see Stokey and Lucas with Prescott 1989, ch.15, e.g., for a discussion).
} 


$$
\text { s.t. } \int_{S}\left[\mathbf{p}(s) \mathbf{c}^{i}(s)-w(s) h^{i}(s)-\mathbf{p}(s) \mathbf{e}^{i}-\Delta^{i}\right] d s \leq 0
$$

(ii) given $[\mathbf{p}(s), w(s)], \forall k n^{k}(s)$ solves

$$
\Pi^{k}=\max _{n^{k}(s)} \int_{S}\left\{\mathbf{p}(s) \mathbf{f}^{k}\left[n^{k}(s)\right]-w(s) n^{k}(s)\right\} d s ;
$$

(iii) $\forall s \in S$,

$$
\begin{aligned}
\sum_{k} n^{k}(s)-\int_{I} h^{i}(s) d i & =0 \\
\int_{I} \mathbf{c}^{i}(s) d i-\sum_{k} \mathbf{f}^{k}\left(n^{k}\right)-\overline{\mathbf{e}} & =0 .
\end{aligned}
$$

Garratt et al. (2002, Theorem 1) show that in this kind of model, every sunspot equilibrium allocation can be supported by prices, when adjusted for probabilities, that are constant across states. ${ }^{8}$ Therefore, in the following, we can set $[\mathbf{p}(s), w(s)]=(\mathbf{p}, w)$ for all $s \in S$. Based on this it is immediate that the solution to the firm problem in (3) is constant across all states - to be more accurate, almost surely with respect to $\pi$, but to ease the presentation we describe results as holding in all states.

Lemma $1 \forall k, n^{k}(s)=n^{k} \forall s \in S$.

Proof. The result follows directly from the strict concavity of $f_{j}^{k}$ in any good $j$ that firm $k$ produces.

Something similar is true for consumers, except that in general we must distinguish between states where they are employed and those where they are not. Let $S_{1}^{i}=\left\{s \in S: h^{i}(s)=1\right\}$ be the set of states where $i$ is employed and $S_{0}^{i}=S \backslash S_{1}^{i}$, where we assume $S_{1}^{i}, S_{0}^{i} \in \Sigma$. Also, let $\ell^{i}=\pi\left(S_{1}^{i}\right)$ be the

\footnotetext{
${ }^{8}$ This is a very intuitive arbitrage-like result: since fundamentals are state-invariant, a good delivered if $s$ occurs better have the same price as a good delivered if $s^{\prime}$ occurs, given $s$ and $s^{\prime}$ occur with equal probability.
} 
probability that $i$ is employed. Then consumer choices are constant across states (again, almost surely) in each of these sets.

Lemma $2 \forall i, \mathbf{c}^{i}(s)=\mathbf{c}_{1}^{i} \forall s \in S_{1}^{i}$ and $\mathbf{c}^{i}(s)=\mathbf{c}_{0}^{i} \forall s \in S_{0}^{i}$.

Proof. Given $[\mathbf{p}(s), w(s)]=(\mathbf{p}, w)$, we can rewrite the consumer problem as

$$
\begin{gathered}
\max \int_{S_{1}} U^{i}\left[\mathbf{c}_{1}^{i}(s), 1\right] d s+\int_{S_{0}} U^{i}\left[\mathbf{c}_{0}^{i}(s), 0\right] d s \\
\text { s.t. } \mathbf{p} \int_{S_{1}} \mathbf{c}^{i}(s) d s+\mathbf{p} \int_{S_{0}} \mathbf{c}^{i}(s) d s-w \ell^{i}-\mathbf{p e}^{i}-\Delta^{i} \leq 0,
\end{gathered}
$$

where the maximization is over the sets $S_{1}^{i}$ and $S_{0}^{i}$, as well as $\mathbf{c}_{1}^{i}(s)$, which is consumption in state $s \in S_{1}$, and $\mathbf{c}_{0}^{i}(s)$, which is consumption in state $s \in S_{0}$. The result now follows from the strict concavity of $U^{i}$.

Lemma 2 implies (6) can be reduced to $\ell^{i} U^{i}\left(\mathbf{c}_{1}^{i}, 1\right)+\left(1-\ell^{i}\right) U^{i}\left(\mathbf{c}_{0}^{i}, 0\right)$, and (7) to $\ell^{i} \mathbf{p} \mathbf{c}_{1}^{i}+\left(1-\ell^{i}\right) \mathbf{p} \mathbf{c}_{0}^{i}-w \ell^{i}-x^{i} \leq 0$, where $x^{i}=\mathbf{p} \mathbf{e}^{i}+\Delta^{i}$ is non-labor income or wealth. ${ }^{9}$ Clearly, $i$ cares only about the probability with which he works, $\ell^{i}=\pi\left(S_{1}^{i}\right)$, and not about which states are in $S_{1}^{i}$. Also, note that while $\mathbf{c}_{1}^{i}$ does not equal $\mathbf{c}_{0}^{i}$, in general, it does for some specifications. The following result says that if some commodities enter $U^{i}$ separably from $h$, then the demand for these commodities is the same whether or not $i$ is employed. As a special case, if $U^{i}\left(\mathbf{c}^{i}, h\right)=u^{i}\left(\mathbf{c}^{i}\right)+v^{i}(h)$, then $\mathbf{c}_{1}^{i}=\mathbf{c}_{0}^{i}$.

Lemma 3 Suppose we can partition $\mathbf{c}^{i}=\left(\hat{\mathbf{c}}^{i}, \tilde{\mathbf{c}}^{i}\right)$, so that $U^{i}\left(\mathbf{c}^{i}, h\right)=u^{i}\left(\tilde{\mathbf{c}}^{i}\right)+$ $v^{i}\left(\hat{\mathbf{c}}^{i}, h\right)$. Then $\tilde{\mathbf{c}}^{i}(s)=\tilde{\mathbf{c}}^{i} \forall s \in S$.

Proof. The result follows directly from strict concavity.

\footnotetext{
${ }^{9}$ Implicitly, $x^{i}$ depends on $\mathbf{p}$ directly, and on $\Delta^{i}$ which also depends on $\mathbf{p}$.
} 
Given Lemmas 1 and 2, we can summarize the decisions of all firms by $\left(n^{k}\right)$ and of all consumers by $\left(\mathbf{c}_{1}^{i}, \mathbf{c}_{0}^{i}, \ell^{i}\right)$, which allows us to present a much simpler definition of equilibrium. ${ }^{10}$ First, one more piece of notation: let $I_{1}(s)=\left\{i \in I: h^{i}(s)=1\right\}=\left\{i \in I: s \in S_{1}^{i}\right\}$ be the set of agents who are employed, and $I_{0}(s)=I \backslash I_{1}(s)$ the set who are unemployed, for each state $s$, where we assume $I_{1}(s), I_{0}(s) \in \Omega$. We need to include $I_{1}(s)$ in our list of equilibrium objects because we need to know who is working (and not just the measure of the set of employed agents) in each state.

Definition 2 An equilibrium is now a list $\left\{\left(\mathbf{c}_{1}^{i}, \mathbf{c}_{0}^{i}, \ell^{i}\right),\left(n^{k}\right),(\mathbf{p}, w), I_{1}(s)\right\}$ satisfying:

(i) given $(\mathbf{p}, w), \forall i\left(\mathbf{c}_{1}^{i}, \mathbf{c}_{0}^{i}, \ell^{i}\right)$ solves

$$
\begin{gathered}
W^{i}=\max _{\mathbf{c}_{1}^{i}, \mathbf{c}_{0}^{i}, \ell^{i}}\left\{\ell^{i} U^{i}\left(\mathbf{c}_{1}^{i}, 1\right)+\left(1-\ell^{i}\right) U^{i}\left(\mathbf{c}_{0}^{i}, 0\right)\right\} \\
\text { s.t. } \ell^{i} \mathbf{p} \mathbf{c}_{1}^{i}+\left(1-\ell^{i}\right) \mathbf{p} \mathbf{c}_{0}^{i}-w \ell^{i}-x^{i} \leq 0 ;
\end{gathered}
$$

(ii) given $(\mathbf{p}, w), \forall k n^{k}$ solves

$$
\Pi^{k}=\max _{n^{k}}\left\{\mathbf{p f}^{k}\left(n^{k}\right)-w n^{k}\right\}
$$

(iii) $\forall s \in S$,

$$
\begin{gathered}
\sum_{k} n^{k}=\alpha\left[I_{1}(s)\right] \\
\overline{\mathbf{e}}+\sum_{k} \mathbf{f}^{k}\left(n^{k}\right)=\int_{I_{1}(s)} \mathbf{c}_{1}^{i} d i+\int_{I_{0}(s)} \mathbf{c}_{0}^{i} d i
\end{gathered}
$$

(iv) $\forall i$

$$
\ell^{i}=\pi\left\{s: I_{1}(s) \ni i\right\}
$$

\footnotetext{
${ }^{10} \mathrm{It}$ is simpler mainly because the firm problem has been reduced to choosing $n^{k}$ and the consumer problem to choosing $\left(\mathbf{c}_{1}^{i}, \mathbf{c}_{0}^{i}, \ell^{i}\right)$, which are finite-dimensional objects (instead of functions of $s$ ).
} 
Labor demand on the left side of (11) is constant across states; hence so is labor supply on the right side. This means we have to construct $I_{1}(s)$ so that the same measure of workers are employed for all $s$, and the last consistency condition says that we need to do so in such a way that each agent $i$ is working with his chosen probability $\ell^{i}$. For example, suppose we have homogeneous consumers, so $\ell^{i}=\bar{\ell}$ for all $i$. Then in equilibrium $\sum_{k} n^{k}=\bar{\ell}$. We have to construct $I_{1}(s)$ so that a fraction $\bar{\ell}$ of consumers are working in every state, and they are all working in a fraction $\bar{\ell}$ of the states.

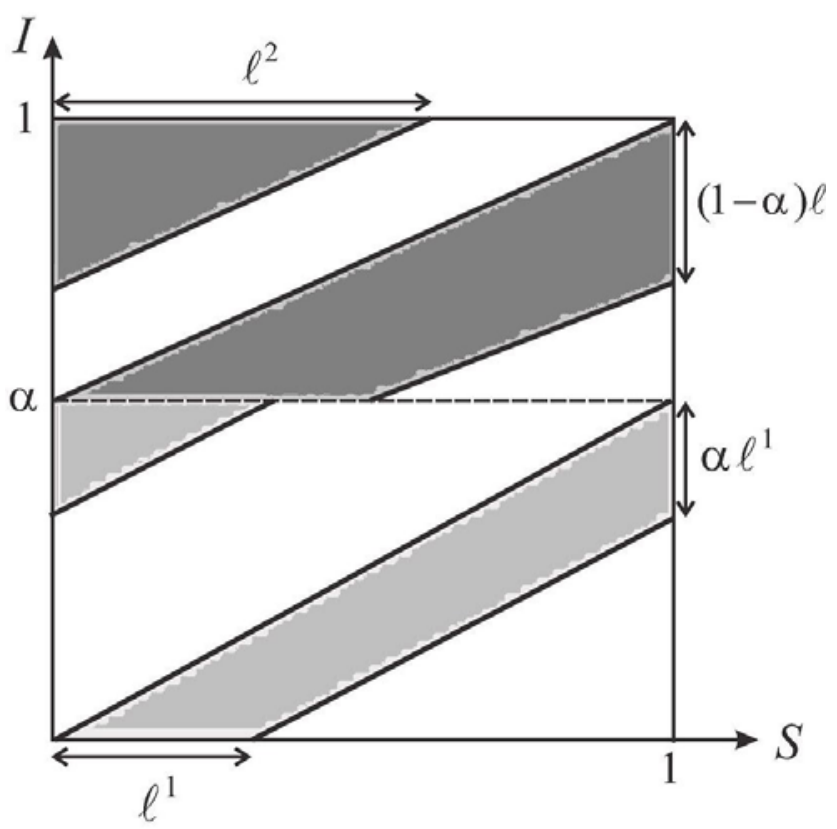

Figure 1: Set of agents working in each state.

This type of construction can be done by generalizing the method in Shell and Wright (1993). Consider an example with two types of consumers: the set $[0, \alpha]$ are type 1 and wish to work with probability $\ell^{1}$, while the rest are type 2 and wish to work with probability $\ell^{2}$. Set $S_{1}^{i}=\left[\frac{i}{\alpha}, \frac{i}{\alpha}+\ell^{1}\right]$ modulo 
$\ell^{1}$ for $i \in[0, \alpha]$, and $S_{1}^{i}=\left[\frac{i-\alpha}{1-\alpha}, \frac{i-\alpha}{1-\alpha}+\ell^{2}\right]$ modulo $\ell^{2}$ for $i \in(\alpha, 1]$. Figure 1 shows as the shaded area pairs $(s, i)$ such that $i$ is employed in state $s$. Then every consumer is working with the desired probability, and the measure of $I_{1}(s)$ (total employment) is $\alpha \ell^{1}+(1-\alpha) \ell^{2}$ for all $s$. However, there is a simpler alternative when we have a continuum of consumers. If type $\tau$ want to work with probability $\ell^{\tau}$, in each state $s$ set $h^{\tau}=1$ with probability $\ell^{\tau}$, and by the law of large numbers a measure $\ell^{\tau}$ will be working in each state. $^{11}$

\subsection{Equilibrium: Properties}

Now that we have defined equilibrium, we provide some substantive results.

First note that (11)-(12) can be rewritten after some manipulation as

$$
\begin{gathered}
\sum_{k} n^{k}-\int_{I} \ell^{i} d i=0 \\
\int_{I}\left[\ell^{i} \mathbf{c}_{1}^{i}+\left(1-\ell^{i}\right) \mathbf{c}_{0}^{i}\right] d i-\overline{\mathbf{e}}-\sum_{k} \mathbf{f}^{k}\left(n^{k}\right)=0
\end{gathered}
$$

This suggests that our economy has a reduced form that looks like a model with no sunspots, where agents simply trade a probability of working $\ell^{i}$ for wages, and use it to buy consumption, in the spirit of lottery models such as Rogerson (1986).

A particularly simple case is the one where $U^{i}\left(\mathbf{c}^{i}, h^{i}\right)=u^{i}\left(\mathbf{c}^{i}\right)+v^{i}\left(h^{i}\right)$ is separable in $h$, since then Lemma 3 implies $\mathbf{c}_{1}^{i}=\mathbf{c}_{0}^{i}=\mathbf{c}^{i}$. Without loss in generality, set $v^{i}(1)=0$ and $v^{i}(0)=A>0$. Then the consumer problem can be simplified further to

$$
W^{i}=\max _{\mathbf{c}^{i}, \ell^{i}}\left\{u^{i}\left(\mathbf{c}^{i}\right)-A^{i} \ell^{i}\right\}
$$

\footnotetext{
${ }^{11}$ Again, we do not need the law of large numbers for sunspot equilibrium, as one does for lottery equilibrium, but it does simplify things slightly.
} 


$$
\text { s.t. } \mathbf{p c}^{i}-w \ell^{i}-x^{i} \leq 0 \text {. }
$$

In this case, it is as if the consumer had a utility function that was linear in $\ell^{i}$. Assuming an interior solution, the first-order condition for any commodity $j$ is $u_{j}^{i}\left(\mathbf{c}^{i}\right)=A^{i} p_{j} / w$. This immediately implies $\partial c_{j} / \partial x^{i}=0, \partial c_{j} / \partial p_{j}=$ $A^{i} p_{j} / w u_{j j}^{i}<0$, and $\partial W^{i} / \partial x^{i}=A^{i} / w$. Hence, in this very special case it is obvious that wealth effects are 0 , demand curves slope downward, and the indirect utility function is linear in $x^{i}$.

We now show these results are general, in the sense that they do not require $U^{i}$ to be separable in $h^{i}$; all we need is interiority.

Proposition 1 Suppose $\ell^{i} \in(0,1)$ and $w-\mathbf{p c}_{1}^{i}+\mathbf{p c}_{0}^{i} \neq 0$. Then $\partial \mathbf{c}_{h j}^{i} / \partial x^{i}=$ $0 \forall i, j$, and $h=0,1$.

Proof. Consider the Lagrangian

$$
\begin{aligned}
W= & \ell U\left(\mathbf{c}_{1}, 1\right)+(1-\ell) U\left(\mathbf{c}_{0}, 0\right) \\
& +\lambda\left[w \ell+x-\ell \mathbf{p} \mathbf{c}_{1}-(1-\ell) \mathbf{p} \mathbf{c}_{0}\right]
\end{aligned}
$$

where $\lambda>0$ is the multiplier, and we leave off the index $i$ where there is no risk of confusion. In the Appendix we show that there is a unique solution to this problem, and assuming $\ell \in(0,1)$ it uniquely satisfies the first-order conditions:

$$
\begin{aligned}
\mathbf{c}_{1} & : U_{j}\left(\mathbf{c}_{1}, 1\right)-\lambda p_{j}=0 \forall j \\
\mathbf{c}_{0} & : \quad U_{j}\left(\mathbf{c}_{0}, 0\right)-\lambda p_{j}=0 \forall j \\
\ell & : \quad U\left(\mathbf{c}_{1}, 1\right)-U\left(\mathbf{c}_{0}, 0\right)+\lambda\left(w-\mathbf{p} \mathbf{c}_{1}+\mathbf{p} \mathbf{c}_{0}\right)=0 \\
\lambda & : \quad w \ell+x-\ell \mathbf{p} \mathbf{c}_{1}-(1-\ell) \mathbf{p} \mathbf{c}_{0}=0
\end{aligned}
$$


Notice that $x$ does not appear in (19)-(21). By the Implicit Function Theorem, these $2 J+1$ equations determine $\left(\mathbf{c}_{1}, \mathbf{c}_{0}, \lambda\right)$ independently of $x$, as long as

$$
\left[\begin{array}{ccc}
\mathbf{H}_{1} & \mathbf{0} & -\mathbf{p}^{T} \\
\mathbf{0} & \mathbf{H}_{0} & -\mathbf{p}^{T} \\
\mathbf{0} & \mathbf{0} & w-\mathbf{p c}_{1}+\mathbf{p} \mathbf{c}_{0}
\end{array}\right]
$$

is nonsingular, where $\mathbf{H}_{h}$ is the Hessian matrix with $(i, j)$ term $U_{i j}\left(c_{h}, h\right)$ and $\mathbf{p}^{T}$ is the transpose. By strict concavity of $U,\left|\mathbf{H}_{h}\right|<0$ and so nonsingularity is equivalent to $w-\mathbf{p} \mathbf{c}_{1}+\mathbf{p c}_{0} \neq 0$.

In the above result we rule out the possibility $w-\mathbf{p c}_{1}+\mathbf{p c}_{0}=0$, which is equivalent to $U\left(\mathbf{c}_{1}, 1\right)=U\left(\mathbf{c}_{0}, 0\right)$ by (21). It is well known that this possibility arises for a very special utility function in the case of 1 good, $U(c, h)=u[c+v(h)] .{ }^{12}$ More generally, consider $U(\mathbf{c}, h)=u\left[c_{J}+v\left(\mathbf{c}^{-J}, h\right)\right]$ where $\mathbf{c}^{-J}=\left(c_{1}, \ldots c_{J-1}\right)$ (i.e. a concave transformation of a utility function that is linear in some good, where we assume without loss in generality that good is $\left.c_{J}\right)$. The first order conditions (19)-(22) still hold, but now notice that for good $J,(19)$ and $(20)$ tell us $u^{\prime}\left[c_{1 J}+v\left(\mathbf{c}_{1}^{-J}, 1\right)\right]=u^{\prime}\left[c_{0 J}+v\left(\mathbf{c}_{0}^{-J}, 0\right)\right]$, and so

$$
c_{1 J}+v\left(\mathbf{c}_{1}^{-J}, 1\right)=c_{0 J}+v\left(\mathbf{c}_{0}^{-J}, 0\right)
$$

Hence in this case $U\left(\mathbf{c}_{1}, 1\right)=U\left(\mathbf{c}_{0}, 0\right)$ and $(21)$ implies $w-\mathbf{p} \mathbf{c}_{1}+\mathbf{p c}_{0}=0$. Now we cannot solve (19)-(21) for $\left(\mathbf{c}_{1}, \mathbf{c}_{0}, \lambda\right)$ independently of $x$. Indeed, (22) now implies $\mathbf{p c}_{0}=x$. However, now (19)-(20) tell us that, for $j \neq J$, we have

$$
\begin{aligned}
u^{\prime}\left[c_{1 J}+v\left(\mathbf{c}_{1}, 1\right)\right] v_{j}\left(\mathbf{c}_{1}, 1\right) & =\lambda p_{j} \Rightarrow v_{j}\left(\mathbf{c}_{1}, 1\right)=p_{j} / p_{J}, j=1,2 \ldots J-1 \\
u^{\prime}\left[c_{0 J}+v\left(\mathbf{c}_{0}, 0\right)\right] v_{j}\left(\mathbf{c}_{0}, 0\right) & =\lambda p j \Rightarrow v_{j}\left(\mathbf{c}_{0}, 0\right)=p_{j} / p_{J}, j=1,2 \ldots J-1
\end{aligned}
$$

\footnotetext{
${ }^{12}$ See e.g. Cooper (1987) or Rogerson and Wright (1988).
} 
We can solve these for $\mathbf{c}_{1}^{-J}=\mathbf{c}_{1}^{-J}(\mathbf{p})$ and $\mathbf{c}_{0}^{-J}=\mathbf{c}_{0}^{-J}(\mathbf{p})$. Then (23) implies

$$
c_{1 J}-c_{0 J}=v\left[\mathbf{c}_{0}^{-J}(\mathbf{p}), 0\right]-v\left[\mathbf{c}_{1}^{-J}(\mathbf{p}), 1\right]
$$

Now we can conclude two things. First, normalizing $p_{J}=1$ with no loss in generality,

$$
w=\mathbf{p}\left(\mathbf{c}_{1}-\mathbf{c}_{0}\right)=\mathbf{p}^{-J}\left[\mathbf{c}_{1}^{-J}(\mathbf{p})-\mathbf{c}_{0}^{-J}(\mathbf{p})\right]+v\left[\mathbf{c}_{0}^{-J}(\mathbf{p}), 0\right]-v\left[\mathbf{c}_{1}^{-J}(\mathbf{p}), 1\right],
$$

which says that $w$ must be a particular function of $\mathbf{p}$; this is nongeneric in partial equilibrium, but can easily occur in general equilibrium. ${ }^{13}$ Second, from $\mathbf{p c}_{0}=x$ we have

$$
c_{0 J}=x-\mathbf{p}^{-J} \mathbf{c}_{0}^{-J}
$$

in this case it is $c_{0 J}$ that adjusts with $x$ to satisfy the budget equation, and all other variables are independent of $x$.

Given that we have explained how to handle the above special case, to conserve space, from now on we ignore it and simply assume that $w-\mathbf{p c}_{1}^{i}+$ $\mathbf{p c}_{0}^{i} \neq 0$.

Proposition 2 Under the conditions in Proposition 1, $\partial \mathbf{c}_{h j}^{i} / \partial p_{j}<0 \forall i, j$ and $h=0,1$.

Proof. Exercise.

Proposition 3 Under the conditions in Proposition 1, $\partial W^{i} / \partial x^{i}=\lambda^{i}$ is independent of $x^{i}$.

\footnotetext{
${ }^{13}$ Suppose that consumers are homogeneous, $J=1$, and there is a representative firm with $f^{\prime}(0)=\infty$ and $f^{\prime}(1)=0$. Then $\ell=n \in(0,1)$ in equilibrium so $w$ will adjust to satisfy the relevant condition, which with $J=1$ is $w / p=v(0)-v(1)$. That is, the real wage exactly compensates workers for lost leisure.
} 
Proof. We can rearrange (18) as

$$
\begin{aligned}
W= & U\left(\mathbf{c}_{0}, 0\right)+\lambda x-\lambda \mathbf{p} \mathbf{c}_{0} \\
& +\ell\left[U\left(\mathbf{c}_{1}, 1\right)-U\left(\mathbf{c}_{0}, 0\right)+\lambda\left(w-\mathbf{p} \mathbf{c}_{1}+\mathbf{p} \mathbf{c}_{0}\right)\right]
\end{aligned}
$$

By (21), the term in brackets vanishes. From Proposition $1, \mathbf{c}_{0}$ and $\lambda$ are independent of $x$, and the result follows.

We now provide something along the lines of an aggregation theorem. First, start with an economy where consumers have homogeneous preferences and wealth, and consider any equilibrium. Now change the distribution of wealth. Then there is an equilibrium where prices and individual consumption are exactly the same as in the homogeneous-wealth economy, and although individual employment probabilities may be different, aggregate employment is the same. The only requirement is interiority for $\ell^{i}$. As we discuss at the end of this section, this can be guaranteed in a homogeneouswealth economy with certain assumptions on technology. Given interiority for a homogeneous-wealth economy, we can then guarantee interiority with heterogeneous wealth by putting bounds on the extent of the wealth heterogeneity.

Proposition 4 Assume $U^{i}=U \forall i$. Let $\left\{\hat{\mathbf{c}}_{1}, \hat{\mathbf{c}}_{0}, \hat{\ell},\left(\hat{n}^{k}\right), \hat{\mathbf{p}}, \hat{w}\right\}$ be an equilibrium when $x^{i}=\hat{x} \forall i$, satisfying the conditions in Proposition 1. Give each consumer $i$ a transfer $t^{i}$ in units of the numeraire good, so that $x^{i}=\hat{x}+t^{i}$, where $\int_{I} t^{i} d i=0$. Then there exist $\bar{x}>0$ and $\underline{x}<\bar{x}$, constructed in the proof, with the following property: if $x^{i} \in(\underline{x}, \bar{x}) \forall i$, then an equilibrium exists with $\ell^{i} \in(0,1) \forall i$ and it has the same $(\hat{\mathbf{p}}, \hat{w})$ and $\left(\mathbf{c}_{1}^{i}, \mathbf{c}_{0}^{i}\right)=\left(\hat{\mathbf{c}}_{1}, \hat{\mathbf{c}}_{0}\right) \forall i$. Although $\ell^{i}$ may differ with $i, \int_{I} \ell^{i} d i=\hat{\ell}$. 
Proof. In the homogeneous-wealth economy, from the budget equation,

$$
\hat{\ell}=\frac{\hat{\mathbf{p}} \hat{\mathbf{c}}_{0}-\hat{x}}{\hat{w}+\hat{\mathbf{p}} \hat{\mathbf{c}}_{0}-\hat{\mathbf{p}} \hat{\mathbf{c}}_{1}} \in(0,1)
$$

by assumption. Now consider the economy with transfers, and set $(p, w)=$ $(\hat{p}, \hat{w})$. From $(10), n^{k}=\hat{n}^{k}$ and $\Pi^{k}=\hat{\Pi}^{k}$. From Proposition 1, if $\ell^{i}$ is interior $\forall i$ then $\left(\mathbf{c}_{1}^{i}, \mathbf{c}_{0}^{i}\right)=\left(\hat{\mathbf{c}}_{1}, \hat{\mathbf{c}}_{0}\right)$. From the budget equation,

$$
\ell^{i}=\frac{\hat{\mathbf{p}} \hat{\mathbf{c}}_{0}-x^{i}}{\hat{w}+\hat{\mathbf{p}} \hat{\mathbf{c}}_{0}-\hat{\mathbf{p}} \hat{\mathbf{c}}_{1}} .
$$

Integrating across agents, $\int_{I} \ell^{i} d i=\hat{\ell}$. Since all aggregate quantities are the same, markets clear.

It remains to give conditions on the distribution such that $\ell^{i} \in(0,1) \forall i$. Suppose $\hat{w}+\mathbf{p} \hat{\mathbf{c}}_{0}-\mathbf{p} \hat{\mathbf{c}}_{1}>0$. Then

$$
\begin{aligned}
& \ell^{i}>0 \text { iff } x^{i}<\bar{x}=\hat{\mathbf{p}} \hat{\mathbf{c}}_{0} \\
& \ell^{i}<1 \text { iff } x^{i}>\underline{x}=\hat{\mathbf{p}} \hat{\mathbf{c}}_{1}-\hat{w} .
\end{aligned}
$$

Now suppose $\hat{w}+\mathbf{p} \hat{\mathbf{c}}_{0}-\mathbf{p} \hat{\mathbf{c}}_{1}<0$. Then

$$
\begin{aligned}
& \ell^{i}>0 \text { iff } x^{i}>\underline{x}=\hat{\mathbf{p}} \hat{\mathbf{c}}_{0} \\
& \ell^{i}<1 \text { iff } x^{i}<\bar{x}=\hat{\mathbf{p}} \hat{\mathbf{c}}_{1}-\hat{w} .
\end{aligned}
$$

Note that in both cases $\bar{x}>0$ and $\underline{x}<\bar{x}$. As long as $x^{i} \in(\underline{x}, \bar{x}) \forall i$, then $\ell^{i} \in(0,1)$ for all $i$. Note that $x^{i} \in(\underline{x}, \bar{x}) \forall i$ is possible because $\hat{x} \in(\underline{x}, \bar{x})$ by (24). This completes the proof.

In fact, it is easy to generalize Proposition 4 so that it holds without the assumption of homogeneous preferences.

Corollary 1 Suppose there are $T$ consumer types, with $U^{i}=U^{\tau}$ for all $i \in I_{\tau}$, where $\cup_{\tau=1}^{T} I_{\tau}=I$. Suppose there is an equilibrium when $x^{i}=\hat{x}^{\tau}$ 
$\forall i \in I_{\tau}$ satisfying the conditions in Proposition 1. Give each consumer $i$ a transfer $t_{i}$ so that now $x^{i}=\hat{x}^{\tau}+t^{i}$, where $\int_{I_{\tau}} t^{i} d i=0 \forall \tau$. Then there exist $\bar{x}^{\tau}>0$ and $\underline{x}^{\tau}<\bar{x}^{\tau}$ with the following property: if $x^{i} \in\left(\underline{x}^{\tau}, \bar{x}^{\tau}\right) \forall i \in I_{\tau}, \forall \tau$, then an equilibrium exists with $\ell^{i} \in(0,1) \forall i$ and it has the same prices, the same consumption, and the same total employment for every type.

\section{Proof. Exercise.}

These results say that, as long as it is not too disperse, the wealth distribution does not matter for consumption or aggregate employment. When wealth differs across agents, rich agents will work less and poor agents more, but nothing else changes. This is useful in the monetary economy studied below, where it implies that even if agents enter the market with different amounts of money, they exit with the same. To preview how this works, we present an example where we put money in the utility function - although we want to make it perfectly clear that we commit this sin here only for purposes of illustration, and in the next section the value of money is derived from first principles.

Thus, in addition to leisure, there are two goods, $\mathbf{c}=(c, \hat{m})$, called consumption and money. The endowment is $\mathbf{e}^{i}=\left(0, m^{i}\right)$ with $\int m^{i} d i=$ $M$ (here $m$ denotes money brought into the market and $\hat{m}$ money taken out). We normalize the price of money to 1 , so $p$ is the nominal price of consumption. Money cannot be produced, while $c$ is produced according to $c=B n$, so that the real equilibrium wage in units of consumption is $B \cdot{ }^{14}$ Suppose $U(c, \hat{m}, h)=\log c-v(h)+V(\hat{m})$, with $v(1)-v(0)=A>1$. Since $U$ is separable in $h$, we know $c_{0}=c_{1}=c$ and $\hat{m}_{0}=\hat{m}_{1}=\hat{m}$. Hence the

\footnotetext{
${ }^{14}$ The fact this production function is not strictly concave does violate assumptions made above, but this causes no problems, since we can solve the example explicitly.
} 
consumer problem is

$$
\begin{gathered}
W(m)=\max _{c, \hat{m}, \ell}\{\log c+V(\hat{m})-A \ell\} \\
\text { s.t. } c-B \ell-\frac{m-\hat{m}}{p} \leq 0 .
\end{gathered}
$$

Substituting for $\ell$ from the budget constraint at equality,

$$
W(m)=\max _{c, \hat{m}}\left\{\log c+V(\hat{m})-\frac{A}{B}\left(c-\frac{m-\hat{m}}{p}\right)\right\} .
$$

The first-order conditions for an interior solution imply $c=B / A$ and $V^{\prime}(\hat{m})=$ $A / B p$; hence, $c$ and $\hat{m}$ are indeed independent of $m$. Given $c$, the technology implies $n=c / B=1 / A \in(0,1)$. From the budget equation,

$$
\ell=\frac{1}{B}\left(c-\frac{m-\hat{m}}{p}\right)=\frac{1}{A}-\frac{(m-M) V^{\prime}(M)}{A},
$$

after inserting market clearing, $\hat{m}=M$, and $p=A / B V^{\prime}(M)$. Clearly, $\ell$ is decreasing in $m$, and $\ell \in(0,1)$ iff $m \in(\underline{m}, \bar{m})$, where

$$
\underline{m}=M-\frac{A-1}{V^{\prime}(M)} \text { and } \bar{m}=M+\frac{1}{V^{\prime}(M)} .
$$

The previous example assumes separability in $h$. Suppose instead that $U(c, \hat{m}, h)=c^{a}(1+b-h)^{1-a}+V(\hat{m})$, where $0<a<1$ and $0<b<(1-a) / a$. Now we do not have $c_{0}=c_{1}$, although we still have $\hat{m}_{0}=\hat{m}_{1}=\hat{m}$. The consumer problem is

$$
\begin{gathered}
W=\max \left\{\ell c_{1}^{a} b^{1-a}+(1-\ell) c_{0}^{a}(1+b)^{1-a}+V^{\prime}(\hat{m})\right\} \\
\text { s.t. } \ell c_{1}+(1-\ell) c_{0}-B \ell-\frac{m-\hat{m}}{p} \leq 0 .
\end{gathered}
$$

This time, we substitute for $\hat{m}$ and take the first-order conditions:

$$
\begin{aligned}
c_{1} & : a c_{1}^{a-1} b^{1-a}=p V^{\prime}(\hat{m}) \\
c_{0} & : a c_{0}^{a-1}(1+b)^{1-a}=p V^{\prime}(\hat{m}) \\
\ell & : \quad c_{1}^{a} b^{1-a}-c_{0}^{a}(1+b)^{1-a}=p V^{\prime}(\hat{m})\left[c_{1}-c_{0}-B\right]
\end{aligned}
$$


These can be solved for $c_{0}=a b B /(1-a), c_{1}=a(1+b) B /(1-a)$ and $V^{\prime}(\hat{m})=a^{a}(1-a)^{1-a} B^{a-1} / p$. Again, $c_{1}, c_{0}$ and $\hat{m}$ are independent of $m$. Now $n=a(1+b) \in(0,1)$. From the budget equation,

$$
\ell=a(1+b)+(M-m) a^{-a}(1-a)^{a} B^{a} V^{\prime}(M),
$$

after inserting $\hat{m}=M$ and $p$. Hence, $\ell \in(0,1)$ iff $m \in(\underline{m}, \bar{m})$, where

$$
\underline{m}=M-\frac{a^{a} B^{a}(1-a-a b)}{(1-a)^{a} V^{\prime}(M)} \text { and } \bar{m}=M+\frac{a^{1+a}(1+b) B^{a}}{(1-a)^{a} V^{\prime}(M)} .
$$

As one can see, it is easy to solve examples, and to construct $(\underline{m}, \bar{m})$ explicitly.

\subsection{Equilibrium: Existence.}

We close this section with a discussion of existence in the general model. Define excess demand for labor and goods by

$$
\begin{gathered}
\sum_{k} n^{k}-\int_{I} \ell^{i} d i=N(\mathbf{p}, w) \\
\int_{I}\left[\ell^{i} \mathbf{c}_{1}^{i}+\left(1-\ell^{i}\right) \mathbf{c}_{0}^{i}\right] d i-\overline{\mathbf{e}}-\sum_{k} \mathbf{f}^{k}\left(n^{k}\right)=\mathbf{Z}(\mathbf{p}, w)
\end{gathered}
$$

We claim the following is true: (i) As we show in the Appendix, even though $W^{i}$ is not strictly quasi-concave, there is a unique solution to the consumer problem $\left(\mathbf{c}_{1}^{i}, \mathbf{c}_{0}^{i}, \ell^{i}\right)$ and it is a continuous function of $(\mathbf{p}, w)$. It is clear on the firm side, profit maximization determines $n^{k}$ as a continuous function of $(\mathbf{p}, w)$. Hence, excess demand is a continuous function of $(\mathbf{p}, w)$. (ii) It is clear that excess demand is homogeneous of degree 0. (iii) Integrating the budget equations over agents, it is also clear that Walras' Law holds: $w N(\mathbf{p}, w)+\mathbf{p Z}(\mathbf{p}, w)=0$. (iv) Also, $Z(\mathbf{p}, w)$ and $N(\mathbf{p}, w)$ can be bounded below as long as we bound the production function $\mathbf{f}^{k}$ for all $k$. (v) 
$\max \left[Z_{j}\left(\mathbf{p}_{n}, w_{n}\right), N\left(\mathbf{p}_{n}, w_{n}\right)\right] \rightarrow \infty$ for any sequence s.t. $\left(\mathbf{p}_{n}, w_{n}\right) \rightarrow(\mathbf{p}, w)$ with $p_{j}=0$ or $w=0$, as long as $U^{i}$ and $\mathbf{f}^{k}$ are strictly increasing.

Properties (i)-(v) allow us to apply a standard existence result, such as Proposition 17.C.1 in Mas-Collel, Whinston and Green (1995). Basically, by reducing the model to something that resembles a standard GE economy we can show an equilibrium exists using standard methods.

Proposition $\mathbf{5}$ Given the assumptions in the text, $\exists(\mathbf{p}, w)$ such that $\mathbf{Z}(\mathbf{p}, w)=$ $\mathbf{0}$ and $N(\mathbf{p}, w)=0$.

Of course, existence does not guarantee $\ell^{i} \in(0,1)$ for all $i$. Since many of our results about the properties of equilibrium depend on it, it would be good to have some additional conditions to guarantee interiority. One way one might imagine proceeding is to put curvature restrictions on technology. Thus, if we assume that for at least one firm $k$ and good $j, \partial f_{j}^{k}(n) / \partial n \rightarrow \infty$ as $n \rightarrow 0$, then clearly aggregate labor demand satisfies $\ell=\sum_{k} n^{k}>0$ in any equilibrium. And if we assume that for every firm $k$ and good $j$, $\partial f_{j}^{k}(n) / \partial n \rightarrow 0$ as $n \rightarrow \bar{n}^{k}$ where $\sum_{k} \bar{n}^{k} \leq 1$, then $\ell=\sum_{k} n^{k}<1$ in any equilibrium. However, this assumption would contradict property (v) that we used for existence. Therefore, we take a different route.

To illustrate the logic, consider the case with $J=1$ good, $K=1$ firm, and homogeneous consumers. If $n=1$ in equilibrium then the utility of a representative consumer is $U[f(1)+e, 1]$. To rule-out such an equilibrium assume

$$
U[f(1)+e, 1]<U\left[f(1)+e-f^{\prime}(1), 0\right] .
$$

If (28) holds then a consumer would be better off choosing $\ell=0$ instead of $\ell=1$. Condition (28) holds provided $f^{\prime}(1)<\bar{\omega}$, where $\bar{\omega}>0$ solves 
$U[f(1)+e, 1]=U[f(1)+e-\bar{\omega}, 0]$. Hence, in a homogeneous consumer economy, in any equilibrium $\ell \in(0,1)$ provided (28) holds. By Proposition 4 , if wealth is heterogeneous there is still an equilibrium where $\ell^{i} \in(0,1)$ $\forall i$, as long as wealth is not too heterogeneous.

One could generalize this logic to $J$ goods as follows. Define

$$
\hat{W}(\mathbf{c}, 1)=\max _{\mathbf{c}_{1}} U\left(\mathbf{c}_{1}, 1\right) \text { s.t. } \mathbf{p c}_{1}-\mathbf{p f}(1)-\mathbf{e}=0,
$$

where $\mathbf{p}$ is a solution to $\mathbf{c}_{1}=\mathbf{f}(1)+\mathbf{e}$. Define next

$$
\hat{W}\left(\mathbf{c}_{0}, 0\right)=\max _{\mathbf{c}_{0}} U\left(\mathbf{c}_{0}, 0\right) \text { s.t. } \mathbf{p c}_{0}-\mathbf{p f}(1)-\mathbf{e}+\mathbf{p f}^{\prime}(1)=0
$$

To guarantee that $n=1$ is not an equilibrium we can impose $\hat{W}(\mathbf{c}, 1)<$ $\hat{W}(\mathbf{c}, 0)$ for any $p$ such that $\mathbf{c}_{1}=\mathbf{f}(1)+\mathbf{e}$. This simply requires that $\mathbf{p} \mathbf{f}^{\prime}(1)$ is not too big.

\section{Monetary Theory}

\subsection{The LW Model}

We begin with a review of LW, to provide the basic environment, notation, etc. There is a $[0,1]$ continuum of infinite-lived agents. Time is discrete, and each period is divided into two subperiods. In the first subperiod there is a frictionless centralized market CM. In the second subperiod there is a decentralized market DM with two main frictions: a double-coincidence problem detailed below, and anonymity, which precludes private credit arrangements. These frictions make money essential. ${ }^{15}$

\footnotetext{
${ }^{15}$ See Kocherlakota (1998) and Wallace (2001) for formal discussions of essentiality, especially the role of anonymity. We also emphasize that it is not important for the CM and DM to meet sequentially; versions where they meet simultaneously, but agents cannot be at both places at the same time, are developed by Williamson (2005) and Telyukova and Wright (2005).
} 
There is a single consumption good $c$ in the CM. Agents have 0 endowment of this good, but can produce it with technology $c=h$. Assume for now that agents can only make spot trades in the CM - i.e. they cannot move resources across periods except by carrying money between markets - but we argue below that this is really without loss of generality. In the DM there is also one good $q$. Each agent faces the following possibilities in the DM: with probability $\sigma \leq 1 / 2$ he wants to consume and derives utility $u(q)$; with the same probability he has the ability to produce at disutility cost $\psi(q)$; and with probability $1-2 \sigma$ he neither wants to consume nor can produce. Agents who want to consume and those who can produce meet bilaterally and anonymously in the DM, where they trade using money. ${ }^{16}$

Let $q^{*}$ be the quantity that solves $u^{\prime}(q)=\psi^{\prime}(q)$; this is an important benchmark because it is what a planner would choose - i.e. it is the efficient DM production. Agents discount between the $\mathrm{CM}$ and $\mathrm{DM}$ at rate $\beta_{1}$, and between the $\mathrm{DM}$ and the next $\mathrm{CM}$ at rate $\beta_{2}$. Let $\beta=\beta_{1} \beta_{2}$. There is a stock of money that changes according to $M_{+1}=(1+\gamma) M$, where the subscript +1 indicates next period, and new money is injected (or withdrawn if $\gamma<0$ ) via lump sum transfers (or taxes) in the CM. This completes the description of the basic environment.

An agents wealth in the CM is $x^{i}=m^{i}+\gamma M$, but since $\gamma M$ is constant across agents we use $m^{i}$ as the individual state variable. Thus $W(m)$ and $V(m)$ are the value functions in the CM and DM; they are not indexed by

\footnotetext{
${ }^{16}$ No agent can both produce and consume in this presentation of the model, but this is easy to relax. In fact, in LW, all agents can do both, but there are many specialized goods and agents match randomly, so whether one consumes or produces depends on whom one meets and some direct barter meetings are possible. As we learned from Neil Wallace, this random matching specification is equivalent to a model such as the one presented here, with preference and technology shocks, and for our purposes this version is slightly easier to discuss.
} 
$i$ since agents are identical except for their current money balances. Then

$$
\begin{aligned}
& W(m)=\max _{c, h, \hat{m}}\left\{U(c, h)+\beta_{1} V(\hat{m})\right\} \\
& \text { s.t. } p c+\hat{m}-p h-m-\gamma M \leq 0 .
\end{aligned}
$$

The solution in general depends on $m$. Hence, a distribution of $m$ across agents entering the $\mathrm{CM}$ induces a distribution of $\hat{m}$ across agents exiting the $\mathrm{CM}$ and entering the DM. If $U(c, h)$ is linear in either $c$ or $h$, however, LW show that $\hat{m}$ is independent of $m$, so the distribution across agents entering the $\mathrm{DM}$ is degenerate at $\hat{m}=M(1+\gamma)$, and they also show that $W$ is linear with $\partial W / \partial m=1 / p .^{17}$

In the DM, in each match between a consumer and producer, they bargain over the quantity of goods $q$ and amount of money $d$ to swap, according to the generalized Nash solution with bargaining power for the consumer $\theta$. The deal is constrained by $d \leq \hat{m}$. LW show that in equilibrium consumers trade all their money, $d=\hat{m}$, in exchange for $q=q(\hat{m})$, where $q(\cdot)$ is given by the solution to $\beta_{2} \hat{m} / p_{+1}=g(q)$ with

$$
g(q) \equiv \frac{\theta \psi(q) u^{\prime}(q)+(1-\theta) u(q) \psi^{\prime}(q)}{\theta u^{\prime}(q)+(1-\theta) \psi^{\prime}(q)} .
$$

We go into more detail on bargaining in the next subsection, where the derivation of (31) will be clear; for now we simply note that it is the price in the next CM, $p_{+1}$, that is relevant for $q$ in this DM. ${ }^{18}$ Given these results,

\footnotetext{
${ }^{17}$ There are two caveats: the distribution of $m$ across agents in the CM cannot be too disperse, since we need interior solutions; and one has to check that $V$ is strictly concave. LW give assumptions on primitives to guarantee both.

${ }^{18}$ Note that (31) is the solution for $m$ only below a threshold $m^{*}$, while for $m \geq m^{*}$ we have $(d, q)=\left(m^{*}, q^{*}\right)$; LW prove that $m<m^{*}$ in any equilibrium, so we can ignore this detail. Also note that LW consider only the case $\beta_{2}=1$, but this is not crucial.
} 
the DM value function satisfies

$$
\begin{aligned}
V(\hat{m})= & \sigma\left\{u[q(\hat{m})]+\beta_{2} W_{+1}(0)\right\} \\
& +\sigma\left\{-\psi\left[q\left(M_{+1}\right)\right]+\beta_{2} W_{+1}\left(\hat{m}+M_{+1}\right)\right\} \\
& +(1-2 \sigma) \beta_{2} W_{+1}(\hat{m})
\end{aligned}
$$

The model is solved as follows. Given quasi-linearity, say $U=u(c)-h$, substitute $h$ from the CM budget equation into $W$, and take the first-order condition with respect to $\hat{m}$ :

$$
\beta_{1} V^{\prime}(\hat{m})=1 / p
$$

The envelope condition from $(32)$ is $V^{\prime}(\hat{m})=\sigma u^{\prime}(q) q^{\prime}(\hat{m})+(1-\sigma) \beta_{2} / p_{+1}$. Or, since $q^{\prime}(\hat{m})=\beta_{2} / p_{+1} g^{\prime}(q)$ from the bargaining solution,

$$
V^{\prime}(\hat{m})=\frac{\beta_{2}}{p_{+1}}\left[1-\sigma+\sigma \frac{u^{\prime}(q)}{g^{\prime}(q)}\right] .
$$

Combining (33) and (34), we have

$$
\frac{1}{p}=\frac{\beta}{p_{+1}}\left[1-\sigma+\sigma \frac{u^{\prime}(q)}{g^{\prime}(q)}\right] .
$$

Finally, the bargaining solution implies $1 / p_{+1}=g(q) / \beta_{2} M(1+\gamma)$ and (lagging this) $1 / p=g\left(q_{-1}\right) / \beta_{2} M$, so (35) becomes

$$
g\left(q_{-1}\right)=g(q) \frac{\beta}{1+\gamma}\left[1-\sigma+\sigma \frac{u^{\prime}(q)}{g^{\prime}(q)}\right] .
$$

Given a sequence for $\gamma$, any strictly positive and bounded solution to this difference equation in $q$ constitutes a monetary equilibrium ${ }^{19}$ If $\gamma$ is constant, it makes sense to consider a steady state where all real variables, including $q$,

\footnotetext{
${ }^{19} \mathrm{LW}$ define equilibrium more formally, but it should be clear that, once one has the path for $q$, one can recover all of the other endogenous variables.
} 
are constant. Then the inflation rate is $\gamma$, the real interest rate is $\rho$ defined by $\beta=1 /(1+\rho)$, and the nominal interest rate is $i=(1+\rho)(1+\gamma)-1$. Now (36) reduces to

$$
1+\frac{i}{\sigma}=\frac{u^{\prime}(q)}{g^{\prime}(q)}
$$

This is the basic model. ${ }^{20}$

We close this section by arguing that, in any equilibrium, the assumption of only spot trades in the $\mathrm{CM}$ is not restrictive. First note that no trades can be made for delivery in any meeting of a future DM, since in this market meetings are anonymous (hence, anyone who was supposed to deliver in this market would simply renege). For similar reasons no trades can be made in the CM contingent on events in any future DM (no one sees what happens to other agents in the DM). So we are left with trades made in the current $\mathrm{CM}$ for delivery in some future CM. But in equilibrium no one partakes of these trades, for the following reason.

Suppose we open a market for Arrow securities that deliver 1 unit of purchasing power (money) in the next CM. Let $W(m, b)$ now be the value function for an agent entering the CM with money $m$ and security holdings $b$, and let $\hat{\mathbf{b}}$ be a vector of assets purchased that period. Note that these Arrow securities are not tangible assets that can be traded bilaterally in the DM - they are simply promises of purchasing power to be delivered in the next CM. Extending our earlier results, one can show that $(\hat{m}, \hat{\mathbf{b}})$ is independent of $(m, b)$. Hence, all agents choose the same portfolio, and the market clears at $\hat{\mathbf{b}}=0$. Therefore, we can shut down these asset markets.

\footnotetext{
${ }^{20}$ We do not dwell on substantive results here, except to mention the following. Under standard assumptions, a monetary equilibrium exists iff $i \geq 0$. For $i>0, q<q^{*}$, so equilibrium is inefficient. Welfare is maximized at the Friedman Rule, $i=0$, but if $\theta<1$ then we have $q<q^{*}$ even at $i=0$.
} 


\subsection{A New Model}

We now consider a model similar to LW, except for two main differences: instead of quasi-linearity, we allow a general utility function; and we assume indivisible labor. We also introduce some other extensions, such a general vector of consumption goods $\mathbf{c}$, an arbitrary endowment $\mathbf{e}$ that may differ over time, profit-maximizing firms, and so on, but we continue to assume homogeneous preferences for now: $U^{i}=U(\mathbf{c}, h)$ for all $i$. The key assumption is indivisible labor, and given this, we consider sunspot equilibria. As in the previous section, we assume only spot trades, but argue below that this is again without loss in generality.

In terms of state-contingent commodities, the CM consumer problem is

$$
\begin{gathered}
W^{i}=\max _{\mathbf{c}^{i}(s), h^{i}(s), \hat{m}^{i}(s)} \int_{S}\left\{U\left[\mathbf{c}^{i}(s), h^{i}(s)\right]+\beta_{1} V^{i}\left[\hat{m}^{i}(s)\right]\right\} d s \\
\text { s.t. } \int_{S}\left[\mathbf{p}(s) \mathbf{c}^{i}(s)+\hat{m}^{i}(s)-w(s) h^{i}(s)-\mathbf{p}(s) \mathbf{e}^{i}-m^{i}-\gamma M-\Delta^{i}\right] d s \leq 0 .
\end{gathered}
$$

This is formally equivalent to the consumer problem in Definition 1, even though money is not a standard commodity here. ${ }^{21}$ Hence we can focus on equilibria with $[\mathbf{p}(s), w(s)]=(\mathbf{p}, w)$. Then we know $\left[\mathbf{c}^{i}(s), \hat{m}^{i}(s)\right]=\left(\mathbf{c}_{1}^{i}, \hat{m}_{1}^{i}\right)$ for all $s$ such that $h^{i}(s)=1$ and $\left[\mathbf{c}^{i}(s), \hat{m}^{i}(s)\right]=\left(\mathbf{c}_{0}^{i}, \hat{m}_{0}^{i}\right)$ for all $s$ such that $h^{i}(s)=0$ by Lemma 1 and Lemma 2. Also, given $\hat{m}^{i}$ and $h^{i}$ enter separably, we know $\hat{m}_{1}^{i}=\hat{m}_{0}^{i}=\hat{m}^{i}$ by Lemma 3 .

Therefore the problem reduces to

$$
\begin{gathered}
W^{i}\left(x^{i}\right)=\max _{\mathbf{c}_{1}^{i}, \mathbf{c}_{0}^{i}, \ell^{i}, \hat{m}^{i}}\left\{\ell^{i} U\left(\mathbf{c}_{1}^{i}, 1\right)+\left(1-\ell^{i}\right) U\left(\mathbf{c}_{0}^{i}, 0\right)+\beta_{1} V^{i}\left(\hat{m}^{i}\right)\right\} \\
\text { s.t. } \ell^{i} \mathbf{p} \mathbf{c}_{1}^{i}+\left(1-\ell^{i}\right) \mathbf{p} \mathbf{c}_{0}^{i}+\hat{m}^{i}-w \ell^{i}-x^{i} \leq 0,
\end{gathered}
$$

\footnotetext{
${ }^{21}$ One detail is that one has to show $V^{i}$ is well behaved - in particular, strictly concave - which can be done following the methods in LW.
} 
where $x^{i}=\mathbf{p e}^{i}+m^{i}+\gamma M+\Delta^{i}$. Assuming an interior solution, the first-order conditions are:

$$
\begin{aligned}
& c_{1 j}^{i} \quad: \quad U_{j}\left(\mathbf{c}_{1}^{i}, 1\right)-\lambda^{i} p_{j}=0 \forall j \\
& c_{0 j}^{i}: U_{j}\left(\mathbf{c}_{0}^{i}, 0\right)-\lambda^{i} p_{j}=0 \forall j \\
& \ell^{i}: U\left(\mathbf{c}_{1}^{i}, 1\right)-U\left(\mathbf{c}_{0}^{i}, 0\right)+\lambda^{i}\left(w+\mathbf{p c}_{0}^{i}-\mathbf{p c}_{1}^{i}\right)=0 \\
& \hat{m}^{i}: \beta_{1} V^{\prime}\left(\hat{m}^{i}\right)-\lambda^{i}=0 \\
& \lambda^{i}: \quad w \ell^{i}+x^{i}-\ell^{i} \mathbf{p} \mathbf{c}_{1}^{i}-\left(1-\ell^{i}\right) \mathbf{p} \mathbf{c}_{0}^{i}-\hat{m}^{i}=0
\end{aligned}
$$

Observe that (38)-(40) constitute $2 J+1$ equations in $2 J+1$ unknowns. Under the assumptions of Proposition 1, and in particular $w-\mathbf{p c}_{1}^{i}+\mathbf{p c}_{0}^{i} \neq 0$, these equations can be solved for $\left(\mathbf{c}_{1}^{i}, \mathbf{c}_{0}^{i}, \lambda^{i}\right)$ independently of $\hat{m}^{i}$ and $\ell^{i}$, as a function of $(\mathbf{p}, w)$ but not $x^{i}$. Because the only way consumers differ here is with respect to $x^{i},\left(\mathbf{c}_{1}^{i}, \mathbf{c}_{0}^{i}, \lambda^{i}\right)=\left(\mathbf{c}_{1}, \mathbf{c}_{0}, \lambda\right)$ is the same for all $i$. Given $\lambda$, (41) can be solved for $\hat{m}^{i}$ independently of $\ell^{i}$, as a function of $(\mathbf{p}, w)$ but not $x^{i}$. Notice that in (41) we did not index $V^{\prime}$ by $i$, implying $\hat{m}^{i}=\hat{m}$ for all $i$; this follows from:

Lemma 4 Under the assumptions of Proposition 1, $W^{i}\left(x^{i}\right)$ and $V^{i}\left(\hat{m}^{i}\right)$ depend on $i$, but $\partial W^{i}\left(x^{i}\right) / \partial x^{i}=\lambda$ and $\partial V^{i}\left(\hat{m}^{i}\right) / \partial \hat{m}^{i}=V^{\prime}\left(\hat{m}^{i}\right)$ do not depend on $i$.

Proof. Consider $W^{i}(x)$. We have

$$
\frac{\partial W^{i}}{\partial x}=\left[U\left(\mathbf{c}_{0}, 0\right)-U\left(\mathbf{c}_{1}, 1\right)+\lambda\left(w+\mathbf{p} \mathbf{c}_{0}-\mathbf{p} \mathbf{c}_{1}\right)\right] \frac{\partial \ell^{i}}{\partial x}+\lambda .
$$

The first term vanishes by (40), so $\partial W^{i} / \partial x^{i}=\lambda$, which is independent of $i$ and $x^{i}$. We show the other result below, after we have described $V^{i}$ in more detail; see (47). 
We formalize the analysis of the first-order conditions up to this point as follows.

Lemma 5 Under the assumptions of Proposition 1, we have $\left(\mathbf{c}_{1}^{i}, \mathbf{c}_{0}^{i}, \hat{m}^{i}, \lambda^{i}\right)=$ $\left(\mathbf{c}_{1}, \mathbf{c}_{0}, \hat{m}, \lambda\right) \forall i$, independent of $x^{i}$.

Proof. Follows from the discussion in the text.

Given $\left(\mathbf{c}_{1}, \mathbf{c}_{0}, \hat{m}, \lambda\right),(42)$ implies

$$
\ell^{i}=\frac{\mathbf{p} \mathbf{c}_{0}+\hat{m}^{i}-x^{i}}{w+\mathbf{p} \mathbf{c}_{0}-\mathbf{p} \mathbf{c}_{1}}=\frac{\mathbf{p} \mathbf{c}_{0}+M-m^{i}-\mathbf{p} \mathbf{e}^{i}-\Delta^{i}}{w+\mathbf{p} \mathbf{c}_{0}-\mathbf{p} \mathbf{c}_{1}},
$$

so $\ell^{i}$ is linearly decreasing in $x^{i}$ and hence $m^{i}$. But aggregate labor supply

$$
\bar{\ell}=\bar{\ell}(\mathbf{p}, w)=\frac{\mathbf{p c}_{0}-\mathbf{p} \overline{\mathbf{e}}-\bar{\Delta}}{w+\mathbf{p c}_{0}-\mathbf{p} \mathbf{c}_{1}}
$$

depends only on average real wealth, $\mathbf{p} \overline{\mathbf{e}}+\bar{\Delta}$. This means that aggregate demand for the $J$ consumption goods,

$$
\mathbf{D}(\mathbf{p}, w)=\bar{\ell}(\mathbf{p}, w) \mathbf{c}_{1}(\mathbf{p}, w)+[1-\bar{\ell}(\mathbf{p}, w)] \mathbf{c}_{0}(\mathbf{p}, w),
$$

does not depend on the wealth distribution, or on monetary considerations at all.

On the firm side, profit maximization determines $n^{k}$ as a function of $(\mathbf{p}, w)$. We can now define market clearing by:

$$
\begin{aligned}
\mathbf{D}(\mathbf{p}, w)-\Sigma_{k} \mathbf{f}^{k}\left[n^{k}(\mathbf{p}, w)\right]-\bar{e} & =0 \\
N(\mathbf{p}, w)-\ell(\mathbf{p}, w) & =0 \\
\hat{m}(\mathbf{p}, w)-M(1+\gamma) & =0
\end{aligned}
$$

There are $J+2$ equations and we only determine $J+1$ prices $(\mathbf{p}, w)$, as we have already normalized the price of money to $1 .^{22}$ Existence of a solution to (45), which can be considered a "spot market equilibrium" or an

\footnotetext{
${ }^{22}$ Walras Law also holds here: integrating the budget equations over agents, if the goods and labor markets clear then the money market clears automatically.
} 
equilibrium in the CM, taking $V^{i}$ as given and well behaved, is established exactly as in Proposition 5. Additionally, notice that the CM equilibrium is determined independently of the value of $q$, which does not appear in the above equations: the model displays a neoclassical dichotomy (see Aruoba and Wright 2003). One implication of this is that monetary policy does not affect the CM, as we discuss further below. ${ }^{23}$

To determine properties of $V^{i}$ we now proceed to the DM, beginning with bargaining. Again we use the generalized Nash solution. Consider a meeting between agents $i$ and $i^{\prime}$ where the former is the consumer and the latter the producer. For the consumer, his payoff is $u(q)+\beta_{2} W_{+1}^{i}\left(x_{+1}^{i}-d\right)$ and his threat point $\beta_{2} W_{+1}^{i}\left(x_{+1}^{i}\right)$, where $x_{+1}^{i}=\mathbf{p}_{+1} \mathbf{e}_{+1}^{i}+\hat{m}^{i}+\gamma_{+1} M_{+1}+\Delta_{+1}^{i}$ is his wealth in the next $\mathrm{CM}$ if he does not trade. For the producer, his payoff is $-\psi(q)+\beta_{2} W_{+1}^{i^{\prime}}\left(x_{+1}^{i^{\prime}}+d\right)$ and his threat point $\beta_{2} W_{+1}^{i^{\prime}}\left(x_{+1}^{i^{\prime}}\right)$. Given that $W_{+1}^{i}$ and $W_{+1}^{i^{\prime}}$ both have slope $\lambda_{+1}$ by Lemma 4 , where $\lambda_{+1}$ is the same for all consumers, the bargaining solution reduces to

$$
\max _{q, d}\left[u(q)-\beta_{2} d \lambda_{+1}\right]^{\theta}\left[\beta_{2} d \lambda_{+1}-\psi(q)\right]^{1-\theta}
$$

s.t. $d \leq \hat{m}^{i}$.

One can show that in any equilibrium the constraint holds with equality, exactly as in LW. Substituting $d=\hat{m}^{i}$ into (46), the first-order condition with respect to $q$ is

$$
\theta\left[-\psi(q)+\beta_{2} \hat{m}^{i} \lambda_{+1}\right] u^{\prime}(q)=(1-\theta)\left[u(q)-\beta_{2} d \hat{m}^{i} \lambda_{+1}\right] \psi^{\prime}(q)
$$

This can be rearranged into $\beta_{2} \hat{m}^{i} \lambda_{+1}=g(q)$, where $g(\cdot)$ is exactly the same as (31) above. Since $\hat{m}^{i}=M_{+1}$ for all $i$, in equilibrium, $q=q\left(M_{+1}\right)$ is the

\footnotetext{
${ }^{23}$ Of course, $M$ and $\gamma$ enter the $\mathrm{CM}$ clearing conditions through $\hat{m}=M+\gamma M$, but $\mathbf{c}_{1}$, $\mathbf{c}_{0}$ and $\bar{\ell}$ are independent of $M$ and $\gamma$.
} 
same in every trade. The DM value function satisfies

$$
\begin{aligned}
V^{i}\left(\hat{m}^{i}\right)= & \sigma\left\{u\left[q\left(\hat{m}^{i}\right)\right]+\beta_{2} W_{+1}\left(x_{+1}^{i}-\hat{m}^{i}\right)\right\} \\
& +\sigma\left\{-\psi(q)+\beta_{2} W_{+1}\left(x_{+1}^{i}+M_{+1}\right)\right\} \\
& +(1-2 \sigma) \beta_{2} W_{+1}\left(x_{+1}^{i}\right) .
\end{aligned}
$$

Notice $V^{i}$ is indexed by $i$, because $x_{+1}^{i}=\mathbf{p}_{+1} \mathbf{e}_{+1}^{i}+\hat{m}^{i}+\gamma_{+1} M_{+1}+\Delta_{+1}^{i}$ can differ across individuals. However, as claimed in Lemma 4, the derivative

$$
\frac{\partial V^{i}}{\partial \hat{m}^{i}}=\sigma u^{\prime}(q) q^{\prime}\left(\hat{m}^{i}\right)+(1-\sigma) \beta_{2} \lambda_{+1}=\left[1-\sigma+\sigma \frac{u^{\prime}(q)}{g^{\prime}(q)}\right] \beta_{2} \lambda_{+1}
$$

does not depend on $i$, where we get $q^{\prime}\left(\hat{m}^{i}\right)=\beta_{2} \lambda_{+1} u^{\prime}(q) / g^{\prime}(q)$ from the bargaining solution. Inserting (47) into (41), we have

$$
\lambda=\beta\left[1-\sigma+\sigma \frac{u^{\prime}(q)}{g^{\prime}(q)}\right] \lambda_{+1}
$$

Using $\beta_{2} \hat{m}^{i} \lambda_{+1}=g(q)$, and market clearing $\hat{m}^{i}=M(1+\gamma)$, (48) becomes

$$
g\left(q_{-1}\right)=g(q) \frac{\beta}{1+\gamma}\left[1-\sigma+\sigma \frac{u^{\prime}(q)}{g^{\prime}(q)}\right] .
$$

Observe that (49) is identical to (36). Hence, in terms of the DM, the new model has exactly the same predictions as LW. Of course the CM differs across the two models, because very different commodities are being traded (and in particular, the new model generates equilibrium unemployment, as we discuss in the next section). Still, it is the case that the assumption of only spot trades in the CM is without loss in generality here. This is perhaps less clear here because, e.g., we have arbitrary endowments across agents, so one might think they would want to borrow or lend; but as long as they are at an interior solution, they would just as soon increase or decrease $\ell^{i}$, and again asset markets are not needed. 
Before closing this section, we discuss the maintained assumption $\ell^{i} \in$ $(0,1)$ for all $i$, using the ideas in Proposition 4. In equilibrium all agents enter each CM with one of three values of $m: m=0$ if they consumed in the previous DM; $m=2 M$ if they produced in the previous DM; and $m=M$ if they did not trade. Hence, from (43), for a given $i, \ell^{i}$ takes on one of three values:

$$
\ell^{i}=\left\{\begin{array}{cl}
\frac{\mathbf{p c}_{0}+M-\mathbf{p} \mathbf{e}^{i}-\Delta^{i}}{w+\mathbf{p} \mathbf{c}_{0}-\mathbf{p} \mathbf{c}_{1}} & \text { if } m=0 \\
\frac{\mathbf{p} \mathbf{c}_{0}-\mathbf{p} \mathbf{e}^{i}-\Delta^{i}}{w+\mathbf{p} \mathbf{c}_{0}-\mathbf{p} \mathbf{c}_{1}} & \text { if } m=M \\
\frac{\mathbf{p c}_{0}-M-\mathbf{p} \mathbf{e}^{i}-\Delta^{i}}{w+\mathbf{p} \mathbf{c}_{0}-\mathbf{p} \mathbf{c}_{1}} & \text { if } m=2 M
\end{array}\right.
$$

As in the proof of Proposition 4 there are two cases: $w+\mathbf{p c}_{0}-\mathbf{p c}_{1}>0$, or, equivalently from (40), $U\left(\mathbf{c}_{0}^{i}, 0\right)>U\left(\mathbf{c}_{1}^{i}, 1\right)$; and $w+\mathbf{p c}_{0}-\mathbf{p c}_{1}<0$, or, equivalently, $U\left(\mathbf{c}_{0}^{i}, 0\right)<U\left(\mathbf{c}_{1}^{i}, 1\right)$. For brevity we present only the former case. $^{24}$ Then, for a given $i,(50)$ implies:

$$
\begin{aligned}
& \ell^{i}>0 \forall i \text { iff } \mathbf{p e}^{i}+\Delta^{i}<\mathbf{p c}_{0}-M \forall i \\
& \ell^{i}<1 \forall i \text { iff } \mathbf{p e}^{i}+\Delta^{i}>M-w+\mathbf{p c}_{1} \forall i
\end{aligned}
$$

Or, to put this in real terms, use the bargaining solution to eliminate $M=$ $g\left(q_{-1}\right) / \beta_{2} \lambda$ and rearrange to get:

$$
\begin{aligned}
& \ell^{i}>0 \forall i \text { iff } g\left(q_{-1}\right)<\beta_{2} \lambda\left(\mathbf{p c}_{0}-\mathbf{p} \mathbf{e}^{i}-\Delta^{i}\right) \equiv \Gamma_{1}^{i} \forall i \\
& \ell^{i}<1 \forall i \text { iff } g\left(q_{-1}\right)<\beta_{2} \lambda\left(\mathbf{p e}^{i}+\Delta^{i}-\mathbf{p c}_{1}+w\right) \equiv \Gamma_{2}^{i} \forall i
\end{aligned}
$$

This yields bounds on $g\left(q_{-1}\right), \Gamma_{1}^{i}$ and $\Gamma_{2}^{i}$, which are independent of monetary considerations - i.e. they take on the same values in the nonmonetary economy where $M=0$ - and are strictly positive iff $\ell^{i} \in(0,1)$ in the nonmonetary economy. If $g\left(q_{-1}\right)<\min \left\{\Gamma_{1}^{i}, \Gamma_{2}^{i}\right\}$ for all $i$, we are done. But it

\footnotetext{
${ }^{24}$ The other case is similar. Again we ignore the special intermediate case $U\left(\mathbf{c}_{0}^{i}, 0\right)=$ $U\left(\mathbf{c}_{1}^{i}, 1\right)$.
} 
is easy to see that $g$ is increasing and $q<q^{*}$ at every date in any equilibrium, where $u^{\prime}\left(q^{*}\right)=\psi^{\prime}\left(q^{*}\right)$, exactly as in LW. Hence it is easy to impose conditions that guarantee interiority. ${ }^{25}$

We summarize the results as follows:

Proposition 6 In the model of this section, $\ell^{i} \in(0,1) \forall i$ as long as $g\left(q^{*}\right)<$ $\min \left\{\Gamma_{1}^{i}, \Gamma_{2}^{i}\right\} \forall i$, where $\Gamma_{1}^{i}$ and $\Gamma_{2}^{i}$ are defined in (53) and (54). Given this, the equilibrium q sequence satisfies (49), which is exactly the same as the LW model.

\section{The Phillips Curve}

The basic version of the LW model dichotomizes: as discussed above, one can solve for the allocations in the CM and DM independently. A consequence of this is that money does not affect aggregate production or consumption in the CM, although it does affect real output since $q$ is a real variable. In particular, the fraction of agents who are unemployed, $1-\bar{\ell}$, is independent of the money growth rate, $\gamma$, and therefore the long-run Phillips curve is vertical. $^{26}$

There are various ways to get around the dichotomy. For instance Aruoba, Waller and Wright (2005) discuss how to proceed by generalizing the technology. Here we proceed by generalizing preferences. A key assumption in the previous section is that utility is additively separable be-

\footnotetext{
${ }^{25}$ Intuitively, we need to have the DM not too important, in the sense that $q$ is not too big, because otherwise the value of money is too high and this either forces some people to $\ell=1$ (those with no money trying to aquire $\hat{m}$ ), or forces some people to $\ell=0$ (those with lots of money trying to spend down to $\hat{m}$ ).

${ }^{26}$ To be accurate, money does affect the distribution of $\ell^{i}$ across agents entering the CM with different $m^{i}$, as indicated by (43); but it has no effect on aggregate employment $\bar{\ell}$.
} 
tween the CM and DM. We now assume the utility of consumption in the $\mathrm{DM}$ is $u(q, \mathbf{c}, h)$. To ease the presentation, we assume there is only one CM consumption good $c$, that the endowment of $c$ is 0 , that $\beta_{1}=1$, and that the CM technology is $c=B h$, so the real wage is $B$ and $x^{i}=m^{i}+\gamma M$. Also, we write CM utility as simply $-v(h)$, with $v(1)=A$ and $v(0)=0$, without is without loss in generality since we already have $c$ in $u(q, c, h){ }^{27}$

As we did earlier, we use $m^{i}$ as the CM state variable. The CM problem in terms of state-contingent commodities is

$$
\begin{aligned}
W(m)= & \max _{c(s), h(s), \hat{m}(s)} \int_{S}\{-A h(s)+V[\hat{m}(s), c(s), h(s)]\} d s \\
\text { s.t. } & \int_{S}[p c(s)+\hat{m}(s)-p B h(s)-m-\gamma M] d s \leq 0,
\end{aligned}
$$

where we have left off the superscript $i$ to save space, but otherwise the only innovation is that $(\hat{m}, c, h)$ is the state variable for $V$, since these are all relevant but predetermined for the DM. The usual logic reduces this problem to

$$
\begin{gathered}
W(m)=\max \ell V\left(\hat{m}_{1}, c_{1}, 1\right)-\ell A+(1-\ell) V\left(\hat{m}_{0}, c_{0}, 0\right) \\
\text { s.t. } \ell p c_{1}+(1-\ell) p c_{0}+\ell \hat{m}_{1}+(1-\ell) \hat{m}_{0}-p B \ell-m-\gamma M \leq 0 .
\end{gathered}
$$

First-order conditions for an interior solution are:

$$
\begin{aligned}
c_{h} & : \quad V_{c}\left(\hat{m}_{h}, c_{h}, h\right)-\lambda p=0, h=0,1 \\
\hat{m}_{h} & : V_{m}\left(\hat{m}_{h}, c_{h}, h\right)-\lambda=0, h=0,1 \\
\ell & : V\left(\hat{m}_{1}, c_{1}, 1\right)-V\left(\hat{m}_{0}, c_{0}, 0\right)-A=\lambda p\left(c_{1}-c_{0}-B+\frac{\hat{m}_{1}-\hat{m}_{0}}{p}\right)(57) \\
\lambda & : p B \ell+m+\gamma M-\ell p c_{1}-(1-\ell) p c_{0}-\ell \hat{m}_{1}-(1-\ell) \hat{m}_{0}=0
\end{aligned}
$$

\footnotetext{
${ }^{27}$ One interpretation is that the CM good $c$ is purchased in the first subperiod but only consumed in the second, after participating in the DM.
} 
Given $V$, (55)-(57) constitute 5 equations that determine $\left(c_{1}, c_{0}, \hat{m}_{1}, \hat{m}_{0}, \lambda p\right)$, independently of $\ell$ or $m$; then (58) determines $\ell$. Notice that we do not get a degenerate distribution, but a two point distribution, of money holdings, since in general $\hat{m}_{1} \neq \hat{m}_{0}$. Also, notice that $W^{\prime}=\lambda$. We still have work ahead of us, however, since we still have to analyze $V .^{28}$

We begin with bargaining. Consider a match where the buyer has some arbitrary $(\hat{m}, c, h)$ and the seller $(\tilde{m}, \tilde{c}, \tilde{h})$. Since $W_{+1}^{\prime}=\lambda_{+1}$, generalizing the analysis in the previous section, the generalized Nash solution is

$$
\max _{q, d}\left[u(q, c, h)-u(0, c, h)-\beta \lambda_{+1} d\right]^{\theta}\left[\beta \lambda_{+1} d-\psi(q)\right]^{1-\theta}
$$

s.t. $d \leq \hat{m}$. Notice the only place $\hat{m}$ enters the problem is through the constraint, and $(\tilde{m}, \tilde{c}, \tilde{h})$ does not enter the problem at all. As before, the constraint $d \leq \hat{m}$ must bind, and the first-order condition with respect to $q$ is:

$$
\theta u_{q}(q, \hat{c}, \hat{h})\left[\beta \lambda_{+1} \hat{m}-\psi(q)\right]=(1-\theta)\left[u(q, \hat{c}, \hat{h})-u(0, \hat{c}, \hat{h})-\beta \lambda_{+1} \hat{m}\right] \psi^{\prime}(q)
$$

This can be rewritten $\beta \lambda_{+1} \hat{m}=g(q, c, h)$, where

$$
g(q, c, h) \equiv \frac{\theta u_{q}(q, c, h) \psi(q)+(1-\theta)[u(q, c, h)-u(0, c, h)] \psi^{\prime}(q)}{\theta u_{q}(q, c, h)+(1-\theta) \psi^{\prime}(q)} .
$$

In general, $q=q\left(\hat{m}, c_{h}, h\right)$ depends on the buyer's entire state vector, although when $\theta=1, g(q, c, h)=\psi(q)$ and $q$ depends only on $\hat{m}$. In any case, there are two values of $q_{h}=q\left(\hat{m}_{h}, c_{h}, h\right)$, in equilibrium, corresponding to $h=1,0$ (consumers who were employed and unemployed).

\footnotetext{
${ }^{28}$ In the previous section we could say a lot about the CM equilibium even before analyzing $V$ because the model dichotomized: recall that we could solve (38)-(40) for $\left(\mathbf{c}_{1}^{i}, \mathbf{c}_{0}^{i}, \lambda^{i}\right)$ independently of $\hat{m}^{i}$. The whole point of this section is to study cases that do not dichotomize.
} 
Given this,

$$
\begin{aligned}
V\left(\hat{m}_{h}, c_{h}, h\right)= & \sigma\left[u\left(q_{h}, c_{h}, h\right)+\beta W_{+1}(0)\right] \\
& +\sigma \mathbb{E}\left[u\left(0, c_{h}, h\right)-\psi(q)+\beta W_{+1}\left(\hat{m}_{h}+d\right)\right] \\
& +(1-2 \sigma)\left[u\left(0, c_{h}, h\right)+\beta W_{+1}\left(\hat{m}_{h}\right)\right]
\end{aligned}
$$

where the expectation in the second term is over $q$ and $d$, which now depend for producers on the type of consumer they meet. Thus,

$$
\begin{aligned}
V_{m} & =\beta \lambda_{+1}\left[1-\sigma+\sigma \frac{u_{q}\left(q_{h}, c_{h}, h\right)}{g_{q}\left(q_{h}, c_{h}, h\right)}\right] \\
V_{c} & =\sigma u_{c}\left(q_{h}, c_{h}, h\right)+(1-\sigma) u_{c}\left(0, c_{h}, h\right)-\sigma \mu\left(q_{h}, c_{h}, h\right),
\end{aligned}
$$

where

$$
\mu\left(q_{h}, c_{h}, h\right) \equiv \frac{u_{q}\left(q_{h}, c_{h}, h\right) g_{c}\left(q_{h}, c_{h}, h\right)}{g_{q}\left(q_{h}, c_{h}, h\right)} .
$$

takes into account the effect of $c$ on $q$ in bargaining. If $\theta=1$, however, producers get 0 surplus from trade, so this effect vanishes and

$$
V_{c}=\sigma u_{c}\left(q_{h}, c_{h}, h\right)+(1-\sigma) u_{c}\left(0, c_{h}, h\right) .
$$

Using (59)-(61) to eliminate $V$ and the bargaining solution to eliminate $\hat{m}_{h},(55)-(57)$ become:

$$
\begin{aligned}
\sigma u_{c}\left(q_{h}, c_{h}, h\right)+(1-\sigma) u_{c}\left(0, c_{h}, h\right)= & \sigma \mu\left(q_{h}, c_{h}, h\right)+\lambda p, h=0,1 \\
\beta \lambda_{+1}\left[1-\sigma+\sigma \frac{u_{q}\left(q_{h}, c_{h}, h\right)}{\psi^{\prime}\left(q_{h}\right)}\right]= & \lambda, h=0,1 \\
\lambda p\left(c_{1}-c_{0}-B\right)+A= & \sigma\left[u\left(q_{1}, c_{1}, 1\right)-u\left(q_{0}, c_{0}, 0\right)\right] \\
& +(1-\sigma)\left[u\left(0, c_{1}, 1\right)-u\left(0, c_{0}, 0\right)\right] \\
& -\frac{\lambda-(1-\sigma) \beta \lambda_{+1}}{\lambda_{+1} \beta}\left[g\left(q_{1}, c_{1}, 1\right)-g\left(q_{0}, c_{0}, 0\right)\right]
\end{aligned}
$$


At this stage we look for a steady state where all real variables including $p \lambda$ are constant, which implies $\lambda / \lambda_{+1}=1+\gamma \cdot{ }^{29}$ Then the above system simplifies to:

$$
\begin{aligned}
\sigma u_{c}\left(q_{h}, c_{h}, h\right)+(1-\sigma) u_{c}\left(0, c_{h}, h\right)= & \sigma \mu\left(q_{h}, c_{h}, h\right)+\lambda p, h=0,1 \\
\frac{u_{q}\left(q_{h}, c_{h}, h\right)}{\psi^{\prime}\left(q_{h}\right)}= & 1+\frac{i}{\sigma}, h=0,1 \\
\lambda p\left(c_{1}-c_{0}-B\right)+A= & \sigma\left[u\left(q_{1}, c_{1}, 1\right)-u\left(q_{0}, c_{0}, 0\right)\right] \\
& +(1-\sigma)\left[u\left(0, c_{1}, 1\right)-u\left(0, c_{0}, 0\right)\right] \\
& -(i+\sigma)\left[g\left(q_{1}, c_{1}, 1\right)-g\left(q_{0}, c_{0}, 0\right)\right]
\end{aligned}
$$

Given $i$, (63)-(65) constitute 5 equations determining $\left(c_{1}, c_{0}, q_{1}, q_{0}, p \lambda\right)$. Then aggregate employment is

$$
\bar{\ell}=\frac{c_{0}}{B+c_{0}-c_{1}}
$$

Finally, inserting the bargaining solution $\beta \lambda_{+1} \hat{m}_{h}=g\left(q_{h}, c_{h}, h\right)$ into $\bar{\ell} \hat{m}_{1}+$ $(1-\bar{\ell}) \hat{m}_{0}=M(1+\gamma)$, we can solve for the marginal value of money

$$
\lambda=\frac{\bar{\ell} g\left(q_{1}, c_{1}, 1\right)+(1-\bar{\ell}) g\left(q_{0}, c_{0}, 0\right)}{\beta M},
$$

which pins down the nominal price level $p$, given we already solved for $p \lambda$. It is obvious that this system does not dichotomize, and hence money affects the CM allocation $\left(c_{1}, c_{0}, \bar{\ell}\right)$.

The model is particularly simple when $u(q, c, h)=u(q, c)$, since in this case $c_{1}=c_{0}=c, \hat{m}_{1}=\hat{m}_{0}=\hat{m}$ and $g(q, c, h)=g(q, c)$. Then (65) immediately yields $\lambda p=A / B$, and $(q, c)$ solve the following versions of

\footnotetext{
${ }^{29}$ Money market clearing requires $\ell \hat{m}_{1}+(1-\ell) \hat{m}_{0}=M(1+\gamma)$. Inserting the bargaining solution, we have $\left[\ell \psi\left(q_{1}\right)+(1-\ell) \psi\left(q_{2}\right)\right]=\beta_{2} \lambda_{+1} M(1+\gamma)$. Hence, $M \lambda_{+1}$ is constant in steady state, and $\lambda$ (which is, after all, the marginal utility of money) falls at the rate at which $M$ grows.
} 
(63)-(64):

$$
\begin{aligned}
\sigma u_{c}(q, c)+(1-\sigma) u_{c}(0, c) & =\sigma \mu(q, c)+\frac{A}{B} \\
u_{q}(q, c) & =\left(1+\frac{i}{\sigma}\right) g_{q}(q, c)
\end{aligned}
$$

Consider the case $\theta=1$. Then

$$
\begin{aligned}
\frac{\partial q}{\partial i} & =\frac{-\left[(1-\sigma) u_{c c}(0, c)+\sigma u_{c c}(q, c)\right] \psi^{\prime}}{\sigma D}<0 \\
\frac{\partial c}{\partial i} & =\frac{u_{c q}(c, q) \psi^{\prime}}{\sigma D} \simeq-u_{c q},
\end{aligned}
$$

where $a \simeq b$ means $a$ and $b$ are equal in sign and

$$
\begin{aligned}
D= & {\left[\sigma u_{c c}(q, c)+(1-\sigma) u_{c c}(0, c)\right] k \psi^{\prime \prime} } \\
& -(1-\sigma) u_{q q}(q, c) u_{c c}(0, c)-\sigma\left[u_{q q}(q, c) u_{c c}(q, c)-u_{q c}(q, c)^{2}\right]<0 .
\end{aligned}
$$

By (66), in the case under consideration, $\bar{\ell}=c / B$. Then the steady-state unemployment rate $1-\bar{\ell}$ depends on $i$ and hence inflation according to

$$
\frac{\partial(1-\bar{\ell})}{\partial i} \simeq-\frac{\partial c}{\partial i} \simeq u_{c q} .
$$

This is extremely intuitive. Inflation is a tax on DM activity, and as such it unambiguously reduces $q$. If $u_{c q}>0$ ( $q$ and $c$ are complements) then inflation also reduces $c$ and hence $\bar{\ell}$. But if $u_{c q}<0$ ( $q$ and $c$ are substitutes) then inflation increases $c$ and hence increases $\bar{\ell}$. In the latter case, inflation causes people to substitute out of DM goods and into CM goods, leading to an increase in CM employment. We get a downward-sloping Phillips curve under simple and natural conditions. Summarizing:

Proposition 7 When $u(q, c, h)=u(q, c)$ and $\theta=1$ the model has a longrun relation between inflation and unemployment that slopes down iff $u_{c q}<$ 0 . 
The case $u(q, c, h)=U(c)+u(q, h)$ is also fairly easy, as it again implies $c_{1}=c_{0}$, although not $\hat{m}_{1}=\hat{m}_{0}$. Furthermore, $g(q, c, h)=g(q, h)$. Letting $u(0,1)=u(0,0)=0$, to reduce notation, (63)-(65) become

$$
\begin{aligned}
U^{\prime}(c)= & \lambda p \\
u_{q}\left(q_{1}, 1\right)= & \left(1+\frac{i}{\sigma}\right) g_{q}\left(q_{1}, 1\right) \\
u_{q}\left(q_{0}, 0\right)= & \left(1+\frac{i}{\sigma}\right) g_{q}\left(q_{0}, 0\right) \\
A-\lambda p B= & \sigma\left[u\left(q_{1}, 1\right)-\left(1+\frac{i}{\sigma}\right) g\left(q_{1}, 1\right)\right] \\
& -\sigma\left[u\left(q_{0}, 0\right)-\left(1+\frac{i}{\sigma}\right) g\left(q_{0}, 0\right)\right]
\end{aligned}
$$

It is easy to derive the following:

$$
\begin{aligned}
\frac{\partial q_{h}}{\partial i} & =\frac{g_{q}\left(q_{h}, h\right)}{\sigma u_{q q}\left(q_{h}, h\right)+(\sigma+i) g_{q q}\left(q_{h}, h\right)} \\
\frac{\partial c}{\partial i} & =\frac{g\left(q_{1}, 1\right)-g\left(q_{0}, 0\right)}{B U^{\prime \prime}(c)} \simeq g\left(q_{0}, 0\right)-g\left(q_{1}, 1\right)
\end{aligned}
$$

Using the fact that $\bar{\ell}=c / B$,

$$
\frac{\partial(1-\bar{\ell})}{\partial i} \simeq g\left(q_{1}, 1\right)-g\left(q_{0}, 0\right),
$$

and unemployment decreases with inflation if $g\left(q_{1}, 1\right)<g\left(q_{0}, 0\right)$.

Consider the case $\theta=1$. Then $g(q, h)=\psi(q)$, and it is easy to show that $g\left(q_{1}, 1\right)<g\left(q_{0}, 0\right)$ iff $q_{1}<q_{0}$ iff $u_{q h}<0 . \partial(1-\bar{\ell}) / \partial i \simeq u_{q h}$. Moreover, when $\theta=1$,

$$
\frac{\partial q_{h}}{\partial i}=\frac{\psi^{\prime}\left(q_{h}\right)}{\sigma u_{q q}\left(q_{h}, h\right)-(\sigma+i) \psi^{\prime}\left(q_{h}\right)}<0 .
$$

These results are also extremely intuitive. Inflation reduces $q$. Then if $u_{q h}>0$ ( $q$ and $h$ are complements, or $q$ and leisure are substitutes) this increases leisure and hence reduces $\bar{\ell}$. But if $u_{c q}<0$ ( $q$ and leisure are complements) then inflation reduces leisure and hence increases $\bar{\ell}$. 
Proposition 8 When $u(q, c, h)=u(q, h)$ and $\theta=1$ the model has a longrun relation between inflation and unemployment that slopes down iff $u_{q h}<$ 0 .

\section{Conclusion}

We have presented a framework within which one can analyze general equilibrium with nonconvexities like indivisible labor (although the analysis also applies to other nonconvexities). In nonconvex economies, randomization can be desirable, and we showed how to support random allocations as equilibria using extrinsic uncertainty, or sunspots. These equilibria have certain interesting and convenient properties, perhaps especially the property that agents act as if they have quasi-linear utility. This means that, assuming interiority, we have zero wealth effects, and so the law of demand must hold, for all of the divisible goods. Also, we can do aggregation, in the sense that within limits the distribution of wealth does not matter.

We then applied the results to monetary economics. We presented an alternative to the framework in Lagos and Wright (2005) - an alternative that generates similar results, but is based on indivisible labor and general utility, rather than quasi-linearity utility. We presented our assumptions in a fairly general way, trying to provide a less restrictive model than in previous related work in monetary economics. Finally, we showed that the model, because it is a monetary model that generates equilibrium unemployment, can be used to discuss the Phillips curve. We think the exercise has taught us a lot about equilibrium models with nonconvexities, and about monetary economics, and we hope that the results will also be useful to others. 


\section{Appendix 1: Second-order conditions}

Here we check the second-order conditions for a strict local maximum to the consumer's problem, assuming $\ell \in(0,1)$. The first-order conditions for an interior solution are given by (19)-(22). The bordered Hessian evaluated at any point where they are satisfied is

$$
\mathbf{B}=\left[\begin{array}{cccc}
0 & -\ell \mathbf{p} & -(1-\ell) \mathbf{p} & w-\mathbf{p} \mathbf{c}_{1}+\mathbf{p} \mathbf{c}_{0} \\
-\ell \mathbf{p}^{T} & \ell \mathbf{H}_{1} & 0 & 0 \\
-(1-\ell) \mathbf{p}^{T} & 0 & (1-\ell) \mathbf{H}_{0} & 0 \\
w-\mathbf{p} \mathbf{c}_{1}+\mathbf{p} \mathbf{c}_{0} & 0 & 0 & 0
\end{array}\right]
$$

For a maximum the last $2 J-1$ leading principal minors $\left|\mathbf{B}_{3}\right|,\left|\mathbf{B}_{4}\right|, \ldots\left|\mathbf{B}_{2 J+2}\right|$ must alternate in sign, with $\left|\mathbf{B}_{2 J+2}\right|<0$. To begin,

$$
\left|\mathbf{B}_{2 J+2}\right|=-\left(w-\mathbf{p c}_{1}+\mathbf{p c}_{0}\right)^{2} \ell(1-\ell)\left|\mathbf{H}_{1}\right|\left|\mathbf{H}_{0}\right|<0,
$$

since $\left|\mathbf{H}_{h}\right|<0$ by the strict concavity of $U$. Second, consider

$$
\mathbf{B}_{1+j}=\ell\left[\begin{array}{cc}
0 & -\mathbf{p}_{j} \\
-\mathbf{p}_{j}^{T} & \mathbf{H}_{1 j}
\end{array}\right]
$$

with $j \in\{2, \ldots J\}$. Here $\mathbf{p}_{j}=\left(p_{1}, \ldots p_{j}\right)$ and $\mathbf{H}_{h j}$ is the submatrix of $\mathbf{H}_{h}$ defined by deleting all but the first $j$ columns and rows. By the strict concavity of $U,\left|\mathbf{B}_{1+j}\right|$ has the same sign as $(-1)^{j}$.

Consider next

$$
\mathbf{B}_{1+J+j}=\left[\begin{array}{ccc}
0 & -\ell \mathbf{p} & -(1-\ell) \mathbf{p}_{j} \\
-\ell \mathbf{p}^{T} & \ell \mathbf{H}_{1} & 0 \\
-(1-\ell) \mathbf{p}_{j}^{T} & 0 & (1-\ell) \mathbf{H}_{0 j}
\end{array}\right]
$$

with $j \leq J$. Then

$$
\left|\mathbf{B}_{1+J+j}\right|=(1-\ell) U_{j j}\left(\mathbf{c}_{0}, 0\right)\left|\mathbf{B}_{J+j}\right|-(1-\ell)^{2} p_{j}^{2} \ell(1-\ell)\left|\mathbf{H}_{1}\right|\left|\mathbf{H}_{0 j-1}\right| .
$$

By induction, $\left|\mathbf{B}_{1+J+j}\right|$ has the same sign as $(-1)^{J+j}$. To see this, note that $\left|\mathbf{B}_{J+2}\right|$ has the same sign as $(-1)^{J+1}$ and $\left|\mathbf{H}_{1}\right|\left|\mathbf{H}_{0 j-1}\right|$ has the same sign as 
$(-1)^{J+j-1}$. Therefore any point that satisfies the first-order conditions is a strict local maximum.

\section{Appendix 2. Global maximum}

Here we use the results in Appendix 1 to show that a solution to the firstorder conditions constitutes the global maximum. We begin by breaking the problem into two steps. First define

$$
\begin{gathered}
\mathcal{V}(\ell)=\max _{\mathbf{c}_{1}, \mathbf{c}_{0}}\left[\ell U\left(\mathbf{c}_{1}, 1\right)+(1-\ell) U\left(\mathbf{c}_{0}, 0\right)\right] \\
\text { s.t. } \ell \mathbf{p} \mathbf{c}_{1}+(1-\ell) \mathbf{p} \mathbf{c}_{0}-\ell w-x \leq 0
\end{gathered}
$$

Since $U$ is strictly concave, this problem has a unique solution $\left[\mathbf{c}_{0}(\ell), \mathbf{c}_{1}(\ell)\right]$. By the Theorem of the Maximum, $\mathcal{V}(\ell)$ is continuous and hence achieves a maximum over $\ell \in[0,1]$.

Suppose there are two local maxima. Then by continuity $\mathcal{V}(\ell)$ also has a local minimum at some $\tilde{\ell} \in(0,1)$. Then $\left[\mathbf{c}_{0}(\tilde{\ell}), \mathbf{c}_{1}(\tilde{\ell}), \tilde{\ell}\right]$ is a saddle-point of the problem in Appendix 1, which contradicts the result that any solution to the first-order conditions is a local maximum. Hence there is a unique

maximizer of $\mathcal{V}(\ell)$, say $\hat{\ell}$, and $\left[\mathbf{c}_{0}(\hat{\ell}), \mathbf{c}_{1}(\hat{\ell}), \hat{\ell}\right]$ is the unique solution to the problem in Appendix 1. 


\section{References}

[1] Aiyagari, S. Rao and Neil Wallace (1991) "Existence of Steady States with Positive Consumption in the Kiyotaki-Wright Model." Review of Economic Studies 58, 901-916.

[2] Aruoba, S. Boragan and Randall Wright (2003) "Search, Money, and Capital: A Neoclassical Dichotomy." J. Money, Credit and Banking 35, 1085-1105.

[3] Aruoba, S. Boragan, Chris Waller and Randall Wright (2005) "Money and Capital." mimeo.

[4] Aumann, Robert (1964) "Markets with a continuum of traders", Econometrica 32, 39-50.

[5] Aumann, Robert (1966) "Existence of competitive equilibria in markets with a continuum of traders", Econometrica 34, 1-17.

[6] Camera, Gabriele and Dean Corbae (1999) "Money and price dispersion", International Economic Review 40, 985-1008.

[7] Christiano, Lawrence and Martin Eichenbaum (1992) "Current Real Business Cycle Theory and Aggregate Labor Market Fluctuations," American Economic Review 82, 430-450.

[8] Cooley, Thomas and Gary D. Hansen (1989) "The Inflation Tax in a Real Business Cycle Model." American Economic Review 79, 733-748.

[9] Cooper, Russel (1987) Wage and employment patterns in labor contracts: Microfoundations and macroeconomic applications, Harwood Academic Publishers. 
[10] Faig, Miquel (2004) "Divisible money in an economy with villages", mimeo.

[11] Garratt, Rod, Todd Keister, Cheng-Zhong Qin and Karl Shell (2002) "Equilibrium prices when the sunspot variable is continuous", Journal of Economic Theory 107, 11-38.

[12] Garratt, Rod, Todd Keister and Karl Shell (2004) "Comparing Sunspot Equilibrium and Lottery Equilibrium Allocations: The Finite Case", International Economic Review 45, 351-386.

[13] Green, Edward J. and Ruilin Zhou (1998) "A rudimentary randommatching model with divisible money and prices", Journal of Economic Theory 81, 252-271.

[14] Hansen, Gary D. (1985) "Indivisible Labor and the Business Cycle." Journal of Monetary Economics 16, 309-327.

[15] Kiyotaki, Nobuhiro and Randall Wright (1989) "On money as a medium of exchange", Journal of Political Economy 97, 927-954.

[16] Kiyotaki, Nobuhiro and Randall Wright (1993) "A Search-Theoretic Approach to Monetary Economics." American Economic Review, 83 63-77.

[17] Kocherlakota, Narayana (1998) "Money is Memory." Journal of Economic Theory 81, 232-251.

[18] Lagos, Ricardo and Randall Wright (2005) "A unified framework for monetary theory and policy analysis", Journal of Political Economy $113,463-84$.

[19] Mas-Collel, Andreu, Michael Whinston and Jerry Green (1995) Microeconomic Theory. Oxford University Press. 
[20] Molico, Miguel (1999) "The distribution of money and prices in search equilibrium", mimeo, forthcoming in International Economic Review.

[21] Rocheteau, Guillaume and Randall Wright ( 2005) "Money in Search Equilibrium, in Competitive Equilibrium, and in Competitive Search Equilibrium." Econometrica 73, 175-202.

[22] Rogerson, Richard (1988) "Indivisible labor, lotteries and equilibrium", Journal of Monetary Economics 21, 3-16.

[23] Rogerson, Richard and Randall Wright (1988) "Involuntary unemployment in economies with efficient risk sharing", Journal of Monetary Economics 22, 501-515.

[24] Shell, Karl and Randall Wright (1993) "Indivisibilities, lotteries, and sunspot equilibria", Economic Theory 3, 1-17.

[25] Shi, Shouyong (1995) "Money and prices: A model of search and bargaining", Journal of Economic Theory 67, 467-496.

[26] Shi, Shouyong (1997) "A divisible search model of fiat money", Econometrica 65, 75-102.

[27] Stockey, Nancy and Robert E. Lucas, Jr. with Edward C. Prescott (1989) Recursive methods in economic dynamics, Harvard University Press, Cambridge.

[28] Taber, Alexander and Neil Wallace (1999) "The Divisibility of Money and Welfare in a Matching Model, "International Economic Review 40, 961-84.

[29] Telyukova, Irina and Randall Wright (2005) "A Model of Money and Credit, with Appilcation to the Credit Card Debt Puzzle," mimeo. 
[30] Trejos, Alberto and Randall Wright (1995) "Search, bargaining, money, and prices", Journal of Political Economy 103, 118-141.

[31] Wallace, Neil (2001) "Whither Monetary Economics?" International Economic Review 42, 847-869.

[32] Williamson, Steve (2005) "Search, Limited Participation, and Monetary Policy," International Economic Review, in press.

[33] Zhou, Ruilin (1999) "Individual and aggregate real balances in a random-matching model", International Economic Review 40, 10091038.

[34] Zhu, Tao (2003) "Existence of a Monetary Steady State in a Matching Model: Indivisible Money." Journal of Economic Theory.

[35] Zhu, Tao (2005) "Existence of a Monetary Steady State in a Matching Model: Divisible Money." Journal of Economic Theory, in press. 